Cite this: Phys. Chem Chem Phys, 2013, 15, 15286

Received 23rd April 2013, Accepted 17th July 2013

DOI: $10.1039 / \mathrm{c} 3 \mathrm{cp} 51741 \mathrm{a}$

www.rsc.org/pccp

\section{Identifying and characterising the different structural length scales in liquids and glasses: an experimental approach}

\author{
Philip S. Salmon* and Anita Zeidler*
}

The structure of several network-forming liquids and glasses is considered, where a focus is placed on the detailed information that is made available by using the method of neutron diffraction with isotope substitution (NDIS). In the case of binary network glass-forming materials with the $\mathrm{MX}_{2}$ stoichiometry (e.g. $\mathrm{GeO}_{2}, \mathrm{GeSe}_{2}, \mathrm{ZnCl}_{2}$ ), two different length scales at distances greater than the nearest-neighbour distance manifest themselves by peaks in the measured diffraction patterns. The network properties are influenced by a competition between the ordering on these "intermediate" and "extended" length scales, which can be manipulated by changing the chemical identity of the atomic constituents or by varying state parameters such as the temperature and pressure. The extended-range ordering, which describes the decay of the pair-correlation functions at large-r, can be represented by making a pole analysis of the Ornstein-Zernike equations, an approach that can also be used to describe the large-r behaviour of the pair-correlation functions for liquid and amorphous metals where packing constraints are important. The first applications are then described of the NDIS method to measure the detailed structure of aerodynamically-levitated laser-heated droplets of "fragile" glass-forming liquid oxides $\left(\mathrm{CaAl}_{2} \mathrm{O}_{4}\right.$ and $\left.\mathrm{CaSiO}_{3}\right)$ at high-temperatures $(\sim 2000 \mathrm{~K})$ and the structure of a "strong" network-forming glass $\left(\mathrm{GeO}_{2}\right)$ under pressures ranging from ambient to $\sim 8 \mathrm{GPa}$. The high-temperature experiments show structural changes on multiple length scales when the oxides are vitrified. The high-pressure experiment offers insight into the density-driven mechanisms of network collapse in $\mathrm{GeO}_{2}$ glass, and parallels are drawn with the high-pressure behaviour of silica glass. Finally, the hydrogen-bonded network of water is considered, where the first application of the method of oxygen NDIS is used to measure the structures of light versus heavy water and a difference of $\simeq 0.5 \%$ is found between the $\mathrm{O}-\mathrm{D}$ and $\mathrm{O}-\mathrm{H}$ intra-molecular bond lengths. The experimental data are best matched by using path integral molecular dynamics simulations with a flexible anharmonic water model, and the results support a competing quantum effects model for water in which its structural and dynamical properties are governed by an offset between intra-molecular and inter-molecular quantum contributions.

\section{Introduction}

Disordered networks are at the heart of many materials of significant scientific and technological importance, ranging from the glasses used for optical fibres and lasers, ${ }^{1-6}$ to water in chemical processes and biological systems ${ }^{7,8}$ It is important to know the structure of these materials in order to understand the physical properties of different network types. It is also necessary to know the structure in a first step to manipulating or designing networks in order to make new materials with the required physical and chemical properties as achieved, for example, by the incorporation of additional chemical species

Department of Physics, University of Bath, Bath BA2 7AY, UK.

E-mail: p.s.salmon@bath.ac.uk, a.zeidler@bath.ac.uk or by processing under high-temperature and/or high-pressure conditions. ${ }^{9}$ The required information is, however, notoriously difficult to access owing to the inherent nature of structural disorder. For instance, an absence of the long-ranged translational periodicity associated with a crystalline phase allows for enhanced flexibility in the bond angles when linking basic structural motifs such as the $\mathrm{SiO}_{4}$ tetrahedra in liquid and glassy silicates.

Unravelling the complexity of structurally disordered materials is therefore a challenging problem, and requires a multidisciplinary approach involving experiment, theory and simulation. From the experimental standpoint, there are several diffraction and spectroscopic techniques for deducing the identity of the local structural motifs, ${ }^{2,6}$ but it is much more difficult to discern the nature of the ordering at distances 
greater than the nearest-neighbour distance. For example, in a neutron or X-ray diffraction experiment on a binary system, the intensity measured as a function of the magnitude of the scattering vector $k$ is represented by three overlapping partial structure factors $S_{\alpha \beta}(k)$, which describe the pair-correlations between atoms of chemical species $\alpha$ and $\beta$. This overlap extends to the corresponding real-space partial pair-distribution functions $g_{\alpha \beta}(r)$, and makes it desirable to devise experiments in order to separate the individual $S_{\alpha \beta}(k)$ functions. Fortunately this is possible because each $S_{\alpha \beta}(k)$ function receives a probedependent weighting factor that can be varied, for example, by changing the incident X-ray energy near the absorption edge of an element in an anomalous X-ray scattering (AXS) experiment ${ }^{10}$ or by substituting the isotope of a given chemical species in a neutron diffraction with isotope substitution (NDIS) experiment. ${ }^{11}$ Information at the partial structure factor level can therefore be obtained by using AXS, ${ }^{12,13}$ by mixing the results obtained from X-ray and neutron diffraction experiments, ${ }^{14-16}$ or by using the NDIS method. ${ }^{17}$

In the following, the focus is on the contribution that NDIS can play in providing information at the partial structure factor level for liquids and glasses. In particular, by matching carefully designed experiments to suitable instrumentation, it is possible to measure accurate diffraction patterns and hence target specific features in the structure of a system. Recent work includes the development of suitable protocols to measure (i) the structure of single droplets of liquid oxides at hightemperatures $(\sim 2000 \mathrm{~K})$ by using the containerless aerodynamiclevitation with laser-heating method, ${ }^{18,19}$ (ii) the structure of glass at high-pressures in the range from ambient to $\sim 8 \mathrm{GPa}$ by employing a Paris-Edinburgh press, ${ }^{20}$ and (iii) the structure of light versus heavy water by using the method of oxygen NDIS where there is a small contrast between the measured diffraction patterns for different isotopically enriched samples. ${ }^{21-23}$

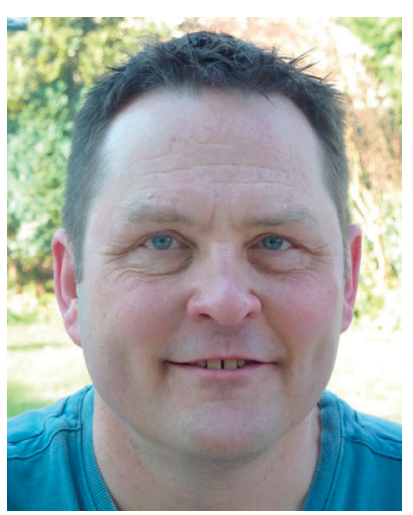

Philip S. Salmon
Phil Salmon was born in Romford in the UK, and is presently a Professor of Physics at the University of Bath. He graduated with a BSc from the University of Bristol in 1981, and completed his PhD in 1985 at the same institution under the supervision of Professor Sir John Enderby FRS. After Bristol, he worked at the University of Exeter as a Research Fellow before joining the University of East Anglia as a "New Blood" Lecturer in 1985.

He moved to Bath as a Senior Lecturer in 1998. His research is focussed on the atomic-scale structure and dynamics of materials, with a particular interest in liquids and glasses. He has benefited from visiting scientist positions at the Institut Laue-Langevin, the University of Oxford, Kyushu University, and the Universite de Montpellier II.
The aim of all of these NDIS experiments is to provide benchmark results to test whether the various theoretical schemes, which need to be employed in the development of accurate predictive models for different classes of materials, contain the right ingredients. For example, how best can transferrable potentials be constructed for use in classical molecular dynamics simulations, ${ }^{24-28}$ and what is the best density functional to use for a given system in first-principles molecular dynamics simulations? ${ }^{29}$ Unlike other atomistic modelling methods such as reverse Monte Carlo (RMC) ${ }^{30,31}$ and Empirical Potential Structure Refinement (EPSR), ${ }^{32,33}$ which use diffraction data to make a three-dimensional structural model, molecular dynamics simulations can explore the dynamical properties of a system and, provided they are suitably constructed, they can be used to predict e.g. the properties of materials under state conditions that extend beyond those for which diffraction data are available. The question as how best to prepare accurate molecular dynamics models of glasses given the use of fast simulated quench-rates is an ongoing source of debate. ${ }^{29,34-36}$

The manuscript is organized as follows. The essential theory for understanding the diffraction results is given in Section 2 and several different classes of system are then discussed. In Section 3, binary network glass-forming materials such as $\mathrm{GeO}_{2}, \mathrm{GeSe}_{2}$ and $\mathrm{ZnCl}_{2}$ are considered where the full set of partial structure factors are now available from NDIS experiments. Two different length scales are found at distances larger than the nearest-neighbour distance and manifest themselves by peaks in the measured diffraction patterns. The so-called first sharp diffraction peak at a scattering vector $k_{\mathrm{FSDP}} \simeq 1-1.5 \AA^{-1}$ is associated with ordering on an intermediate range while the principal peak at $k_{\mathrm{PP}} \simeq 2.0-2.7 \AA^{-1}$ is associated with ordering on an extended range, which can persist to nanometer distances in real-space. The results show that a competition between the ordering on these length scales influences the

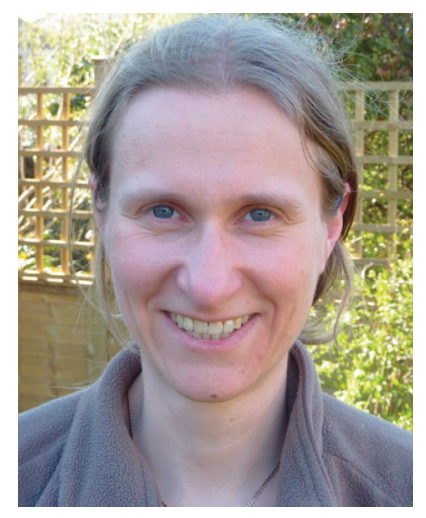

Anita Zeidler
Anita Zeidler was born in Lauchhammer in the former Deutsche Demokratische Republik, and is presently a Research Officer at the University of Bath. She graduated with a Chemiediplom from the Philipps-Universität Marburg in 2004, following a year as an exchange student at the University of Cambridge. She completed her PhD in physics at the University of Bath in 2009. She is extensively involved in developing the methods required to measure and interpret the structure of a wide range of materials, including liquids and glasses under extreme high-pressure and high-temperature conditions, and water in its various phases. 
relative "fragility" 37 of these network glass-forming materials. In Section 4, the structure of liquid and amorphous metals is then considered where packing constraints are important and where the principal peak is an important feature in the measured diffraction patterns. The extended-range ordering, which describes the decay of the measured pair-correlation functions at large- $r$, can be represented for both network glass-forming and densely-packed metallic systems by making a pole analysis of the Ornstein-Zernike equations. ${ }^{38-40}$ In Section 5, the structure of fragile glass-forming liquid oxides is investigated by applying the NDIS method to aerodynamicallylevitated laser-heated droplets of $\mathrm{CaAl}_{2} \mathrm{O}_{4}$ and $\mathrm{CaSiO}_{3} \cdot{ }^{18,19}$ Large structural changes on multiple length scales are observed on vitrification. In Section 6, the first application of the NDIS method to measure the structure of glass in situ at high-pressures is described. In particular, the density-driven mechanisms of structural collapse are investigated in $\mathrm{GeO}_{2}$ glass at pressures up to $\sim 8 \mathrm{GPa}$. Similarities with the behaviour of $\mathrm{SiO}_{2}$ glass are discussed, where there is also a densitydriven transformation from a tetrahedral to an octahedral glass albeit at much higher pressures. In Section 7, the role of competing quantum effects in water is then deliberated in relation to the results obtained from the first application of the oxygen NDIS method to both light and heavy water. Finally, conclusions are drawn in Section 8 and the future outlook is considered.

\section{Diffraction methods}

\subsection{Outline theory}

In a neutron diffraction experiment, the intensity measured as a function of the magnitude of the scattering vector $k$ can be written in terms of the differential scattering cross-section ${ }^{17}$

$$
\mathrm{d} \sigma / \mathrm{d} \Omega=F(k)+P(k)
$$

where the total structure factor, which contains information on the relative positions of pairs of distinct nuclei, is defined by

$$
F(k)=\sum_{\alpha=1}^{n} \sum_{\beta=1}^{n} c_{\alpha} c_{\beta} b_{\alpha} b_{\beta}\left[S_{\alpha \beta}(k)-1\right]
$$

and $P(k)$ contains contributions from the self-scattering by individual nuclei and from inelastic scattering. In eqn (2), $\alpha$ and $\beta$ denote the chemical species, $n$ is the number of different chemical species, while $c_{\alpha}$ and $b_{\alpha}$ represent the atomic fraction and bound coherent neutron scattering length of chemical species $\alpha$, respectively. $S_{\alpha \beta}(k)$ is a so-called Faber-Ziman ${ }^{41}$ partial structure factor which is related to the partial pair-distribution function $g_{\alpha \beta}(r)$ by the Fourier transform relation

$$
g_{\alpha \beta}(r)-1=\frac{1}{2 \pi^{2} \rho r} \int_{0}^{\infty} \mathrm{d} k k\left[S_{\alpha \beta}(k)-1\right] \sin (k r)
$$

where $\rho$ is the atomic number density and $r$ is a distance in real space. The mean coordination number of atoms of type $\beta$, contained in a volume defined by two concentric spheres of radii $r_{i}$ and $r_{j}$ centred on an atom of type $\alpha$, is given by

$$
\bar{n}_{\alpha}^{\beta}=4 \pi \rho c_{\beta} \int_{r_{i}}^{r_{j}} \mathrm{~d} r r^{2} g_{\alpha \beta}(r) .
$$

The real-space information corresponding to $F(k)$ is provided by the total pair-distribution function $G(r)$ which is obtained from the Fourier transform relation

$$
G(r)=\frac{1}{2 \pi^{2} \rho r} \int_{0}^{\infty} \mathrm{d} k k F(k) M(k) \sin (k r)
$$

where $M(k)$ is a modification function which is introduced because a diffractometer can measure over only a finite $k$ range up to a maximum value $k_{\max }$. Provided that sufficiently small $k$-values can be accessed, $M(k)=1$ for $k \leq k_{\max }$ and $M(k)=0$ for $k>k_{\text {max }}$. However, to give smoother $r$-space functions, other expressions are used such as the Lorch ${ }^{42}$ modification function where $M(k)=\sin \left(\pi k / k_{\max }\right) /\left(\pi k / k_{\max }\right)$ for $k \leq k_{\max }$ and $M(k)=0$ for $k>k_{\text {max }} \cdot \uparrow$ To facilitate a comparison between experimental and molecular dynamics results, the reciprocal-space functions constructed from simulations are often Fourier transformed according to eqn (5) with $k_{\max }$ set at the experimental value. If $k_{\max }$ is sufficiently large that $F(k)$ no longer shows structure at high $k$, then $G(r)$ is given by

$$
G(r)=\sum_{\alpha=1}^{n} \sum_{\beta=1}^{n} c_{\alpha} c_{\beta} b_{\alpha} b_{\beta}\left[g_{\alpha \beta}(r)-1\right] .
$$

For $r$-values smaller than the distance of closest approach between the centers of two atoms it follows from the definition of the partial pair-distribution functions that $g_{\alpha \beta}(r)=g_{\alpha \beta}(r \rightarrow 0)=0$ such that $G(r)=G(r \rightarrow 0)=-\langle b\rangle^{2}$ where $\langle b\rangle=\sum_{\alpha=1}^{n} c_{\alpha} b_{\alpha}$ is the mean scattering length.

For a binary system comprising two chemical species denoted by $\mathrm{M}$ and $\mathrm{X}$, it is also instructive to re-write the total structure factor of eqn (2) in terms of the Bhatia-Thornton ${ }^{44}$ number-number, concentration-concentration and numberconcentration partial structure factors, denoted by $S_{\mathrm{NN}}(k)$, $S_{\mathrm{CC}}(k)$ and $S_{\mathrm{NC}}(k)$ respectively, where

$$
\begin{aligned}
F(k)= & \langle b\rangle^{2} S_{\mathrm{NN}}(k)+\left(b_{\mathrm{M}}-b_{\mathrm{X}}\right)^{2} S_{\mathrm{CC}}(k) \\
& +2\langle b\rangle\left(b_{\mathrm{M}}-b_{\mathrm{X}}\right) S_{\mathrm{NC}}(k)-\left(c_{\mathrm{M}} b_{\mathrm{M}}{ }^{2}+c_{\mathrm{X}} b_{\mathrm{X}}{ }^{2}\right) .
\end{aligned}
$$

These partial structure factors $S_{\mathrm{IJ}}(k)(\mathrm{I}, \mathrm{J}=\mathrm{N}, \mathrm{C})$ separate those correlations that give rise to the global non-crystalline structure $S_{\mathrm{NN}}(k)$ from those that describe the chemical ordering $S_{\mathrm{CC}}(k),{ }^{45}$ and their values in the $k \rightarrow 0$ limit are readily linked to the thermodynamic properties of a binary system. $\neq$ The Bhatia-Thornton and Faber-Ziman partial structure factors are related by the expressions

$$
S_{\mathrm{NN}}(k)=c_{\mathrm{M}}^{2} S_{\mathrm{MM}}(k)+c_{\mathrm{X}}{ }^{2} S_{\mathrm{Xx}}(k)+2 c_{\mathrm{M}} c_{\mathrm{X}} S_{\mathrm{MX}}(k)
$$

$\dagger$ The mathematical validity of the Lorch function has been called into question by Soper and Barney. ${ }^{43}$ However, a rigorous derivation, which also gives an analytical expression for the real-space manifestation of this function, is given in ref. 40.

‡ The Bhatia-Thornton formalism can be generalised to systems containing more than two chemical species. ${ }^{46-49}$ 


$$
\begin{aligned}
& S_{\mathrm{CC}}(k)=c_{\mathrm{M}} c_{\mathrm{X}}\left\{1+c_{\mathrm{M}} c_{\mathrm{X}}\left[S_{\mathrm{MM}}(k)+S_{\mathrm{XX}}(k)-2 S_{\mathrm{MX}}(k)\right]\right\} \\
& S_{\mathrm{NC}}(k)=c_{\mathrm{M}} c_{\mathrm{X}}\left\{c_{\mathrm{M}}\left[S_{\mathrm{MM}}(k)-S_{\mathrm{MX}}(k)\right]-c_{\mathrm{X}}\left[S_{\mathrm{XX}}(k)-S_{\mathrm{MX}}(k)\right]\right\} .
\end{aligned}
$$

From eqn (7) it follows that if $b_{\mathrm{M}}=b_{\mathrm{X}}$ the incident neutrons in a diffraction experiment cannot distinguish between the different scattering nuclei and the measured total structure factor gives $S_{\mathrm{NN}}(k)$ directly. The Fourier transform of $S_{\mathrm{NN}}(k)$, the partial pairdistribution function $g_{\mathrm{NN}}(r)$, therefore describes the sites of the scattering nuclei and, since it cannot distinguish between the chemical species that decorate those sites, it gives information on the topological ordering. If $\langle b\rangle=0$, however, the measured total structure factor gives $S_{\mathrm{CC}}(k)$ directly and its Fourier transform, $g_{\mathrm{CC}}(r)$, describes the chemical ordering of the $\mathrm{M}$ and $\mathrm{X}$ atomic species.§ The partial pair-distribution function $g_{\mathrm{CC}}(r)$ will show a positive or negative peak at a given distance when there is a preference for like or unlike neighbours, respectively. The Fourier transform of $S_{\mathrm{NC}}(k)$, namely the partial pairdistribution function $g_{\mathrm{NC}}(r)$, describes the correlation between the sites described by $g_{\mathrm{NN}}(r)$ and their occupancy by a given chemical species.

In the case of an X-ray diffraction experiment, eqn (2) remains valid provided the scattering lengths $b_{\alpha}$ are replaced by the $k$-dependent atomic form factors with dispersion terms $f_{\alpha}(k)$. In order to compensate for this $k$ dependence, the total structure factor is often rewritten as

$$
S(k)-1=\frac{F(k)}{|\langle f(k)\rangle|^{2}}
$$

where $\langle f(k)\rangle=\sum_{\alpha=1}^{n} c_{\alpha} f_{\alpha}(k)$. From eqn (7), it follows that if $f_{\mathrm{M}}(k) \simeq f_{\mathrm{X}}(k)$ (or $\left.b_{\mathrm{M}} \simeq b_{\mathrm{X}}\right)$ then $S(k) \simeq S_{\mathrm{NN}}(k)$ i.e. if both chemical species have the same coherent scattering crosssections then the incident X-rays (or neutrons) will observe the material to be a one component system. The notation $S_{\mathrm{X}}(k)$ and $S_{\mathrm{N}}(k)$ will be used in order to distinguish between the total structure factors measured by X-ray and neutron diffraction, respectively, and a similar notation will be used to distinguish between their corresponding total pair-distribution functions $G_{\mathrm{X}}(r)$ and $G_{\mathrm{N}}(r)$. From eqn (6), it follows that in the case of a neutron diffraction experiment

$$
G_{\mathrm{N}}(r)-1=G(r) /\langle b\rangle^{2} .
$$

The expression for $G_{\mathrm{X}}(r)$ is more complicated because the X-ray form factors in eqn (11) are $k$-dependent. In cases were a clearly defined peak in $G_{\mathrm{X}}(r)$ can be assigned to a specific $g_{\alpha \beta}(r)$ function, however, the corresponding coordination number can still be found by using eqn (4) after Fourier transforming a suitably weighted $k$-space function. ${ }^{51}$

To unravel the complexity of pair-correlation functions associated with the measurement of $F(k)$ for a liquid or glass, it is necessary to obtain information on the partial structure factors. This can be achieved by using the NDIS method ${ }^{11}$

$\S$ The neutron scattering lengths for several isotopes take negative values. It is therefore possible in a neutron diffraction experiment to measure $S_{\mathrm{CC}}(k)$ directly. ${ }^{50}$ where the neutron scattering length of a given chemical species is changed by preparing two or more samples that are identical in every respect, except for the isotopic enrichment of that chemical species. In favourable cases, it is possible to extract the full set of partial structure factors for a binary system directly from the experimental data. ${ }^{11,17}$ In other cases, the use of difference function methods leads to an elimination of selected partial structure factors, thus simplifying the complexity of pair-correlation functions associated with $F(k) .{ }^{17}$

As an illustration, if the diffraction patterns for an $\mathrm{MX}_{2}$ system are measured for $\ell(\geq 3)$ samples that are identical in every respect, except for the isotopic enrichment of one or more of the chemical species, then the equations for the total structure factors can be written in the form

$$
\mathbf{F}=\mathbf{W S}
$$

where $\mathbf{F}$ is a column vector for the total structure factors, $\mathbf{S}$ is a column vector for the partial structure factors, and $\mathbf{W}$ is a $\ell \times 3$ weighting factor matrix with elements defined by eqn (2) or (7). The full set of partial structure factors can then be found by using the method of singular value decomposition (SVD) where $\mathbf{S}=\mathbf{W}^{\dagger} \mathbf{F}$ and $\mathbf{W}^{\dagger}$ is the Moore-Penrose pseudo-inverse of $\mathbf{W}$, which is equal to the inverse matrix $\mathbf{W}^{-1}$ when $\ell=3 .^{52,53}$

\subsection{Instrumentation and data analysis}

Several neutron diffractometers have been designed for work on liquid and amorphous materials, including D4c at the Institut Laue-Langevin, ${ }^{54}$ SANDALS, GEM and NIMROD at the ISIS pulsed neutron source, ${ }^{55-57}$ NOMAD at the Spallation Neutron Source, ${ }^{58}$ and NOVA at the Japan Proton Accelerator Research Complex. ${ }^{59}$ For NDIS experiments, the diffractometer D4c has a proven track record, combining the necessary attributes of a high detector count-rate over a wide $k$-range with a lowbackground signal, an excellent count-rate stability $( \pm 0.01 \%$ over three days), and an ability to re-measure to within $0.020(1) \%$ the diffraction pattern for different mountings of the same sample. ${ }^{23,54}$ It was therefore the diffractometer of choice for the majority of NDIS experiments reported herein.

The corrections that need to be applied to neutron diffraction data for e.g. container scattering, beam attenuation and multiple scattering are generally straight-forward for experiments made under ambient conditions, although inelasticity corrections are an important exception in cases where the masses of the neutron and scattering nuclei are comparable. ${ }^{17}$ It is usual, however, for the corrections to become more difficult for the complex sample environments and scattering geometries encountered in experiments made under extreme conditions of high-pressures or high-temperatures. ${ }^{51,60-62}$ In general, systematic errors that remain in the corrected diffraction patterns can be reduced by using difference function methods where two or more diffraction patterns are subtracted from one another, a technique that can also be used to cancel the worst effects that arise from the inelastic scattering of neutrons by light nuclei. ${ }^{23,63,64}$

For binary systems, our approach is to solve eqn (13) pointby-point, without an application of the constraints that were 
used in the first NDIS experiments. ${ }^{65}$ Uncertainties on the partial structure factors that result from uncertainties on the total structure factors and weighting factor matrix can be assessed by using the SVD method. ${ }^{53}$ The efficacy of the results can be assessed by checking, for example, that the partial structure factors satisfy the required sum-rule and inequality relations, and that they take physically meaningful $k \rightarrow 0$ limiting values. ${ }^{11,44}$ It follows that it should also be possible to construct 3-dimensional models of non-overlapping atoms that are consistent with the measured partial structure factors by using atomistic modelling methods. The merits of this direct approach, in which confidence is assigned to the results obtained from carefully designed experiments, are briefly discussed in Section 3.2 by reference to the case study of liquid and glassy $\mathrm{GeSe}_{2}$.

\section{Network glass-forming materials}

\subsection{Intermediate versus extended-range ordering}

The NDIS method has been used to measure the full set of partial pair-correlation functions for several network glass-forming materials having the $\mathrm{MX}_{2}$ stoichiometry, namely liquid and glassy $\mathrm{GeSe}_{2},{ }^{66-68}$ liquid and glassy $\mathrm{ZnCl}_{2},{ }^{53,69,73}$ and glassy $\mathrm{GeO}_{2} \cdot{ }^{70,71}$ The NDIS method has also been combined with X-ray diffraction to measure the $S_{\alpha \beta}(k)$ functions for $\mathrm{SiO}_{2}$ glass. ${ }^{74}$ In the Angell classification scheme, ${ }^{37} \mathrm{GeO}_{2}$ and $\mathrm{SiO}_{2}$ are regarded as "strong" glass forming liquids whereas $\mathrm{ZnCl}_{2}$ and $\mathrm{GeSe}_{2}$ have a character that is more intermediate between the "strong" and "fragile" extremes (Fig. 1). ${ }^{75}$

The measured Faber-Ziman partial structure factors $S_{\alpha \beta}(k)$ for liquid $\mathrm{GeSe}_{2}$ and for the glasses $\mathrm{GeSe}_{2}, \mathrm{ZnCl}_{2}$ and $\mathrm{GeO}_{2}$ are illustrated in Fig. 2. These reciprocal space functions show a so-called first sharp diffraction peak (FSDP) at $k_{\mathrm{FSDP}} \simeq 1-1.5 \AA^{-1}$ together with a principal peak at a larger scattering vector $k_{\mathrm{PP}} \simeq$ 2.0-2.7 $\AA^{-1}$. The FSDP is associated with intermediate-range order of periodicity given by $2 \pi / k_{\mathrm{FSDP}}$ with a correlation length given by $2 \pi / \Delta k_{\mathrm{FSDP}}$ where $\Delta k_{\mathrm{FSDP}}$ is the full-width at halfmaximum. ${ }^{76-78}$ The largest contribution to the FSDP arises from $\mathrm{M}-\mathrm{M}$ correlations, although the $\mathrm{M}-\mathrm{X}$ and $\mathrm{X}-\mathrm{X}$ correlations also contribute in the cases of $\mathrm{GeO}_{2}$ and $\mathrm{SiO}_{2},{ }^{71,74}$ and it is therefore associated with the ordering of M-centred structural units. In comparison, the principal peak is associated with extended-range order having a periodicity of $2 \pi / k_{\mathrm{PP}}$ with a correlation length given by $2 \pi / \Delta k_{\mathrm{PP}}$ where $\Delta k_{\mathrm{PP}}$ is the full-width at half-maximum. The extended-range order derives its name from the observation that it usually persists to distances well beyond the domain of the FSDP. ${ }^{69,80}$

The measured partial pair-distribution functions $g_{\alpha \beta}(r)$ for liquid and glassy $\mathrm{GeSe}_{2}$, glassy $\mathrm{ZnCl}_{2}$ and glassy $\mathrm{GeO}_{2}$ are illustrated in Fig. 3. Several of the parameters describing the local structure of these materials and liquid $\mathrm{ZnCl}_{2}$ are listed in Table 1. In each case, the structure is built predominantly from tetrahedral $\mathrm{MX}_{4}$ motifs. Corner-sharing tetrahedra predominate

T The periodicity associated with the FSDP can be discerned in the $r$-space functions measured for several network glass-forming systems. ${ }^{78,79}$

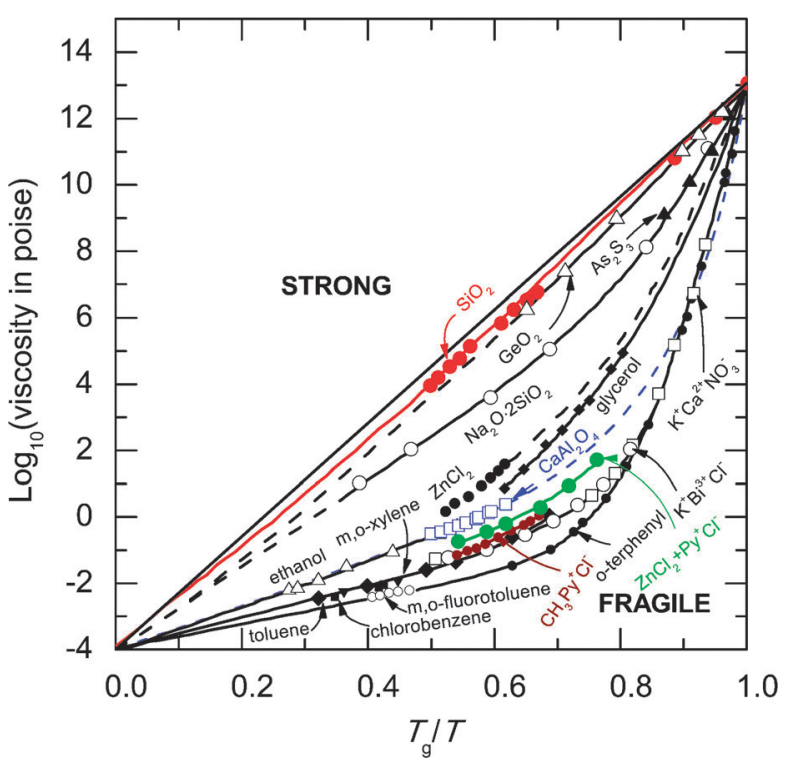

Fig. 1 The logarithm of the liquid viscosity $\eta$ for several glass-forming materials as plotted against $T_{\mathrm{g}} / T$, where $T$ is the absolute temperature and $T_{\mathrm{g}}$ is the glass transition temperature. ${ }^{37}$ The fragility index $m \equiv \mathrm{d} \log _{10} \eta /\left.\mathrm{d}\left(T_{\mathrm{g}} / T\right)\right|_{T=T_{\mathrm{g}}}$ gives the gradient at $T=T_{\mathrm{g}}$ and therefore characterises how steeply the liquid viscosity increases when cooling to form a glass.

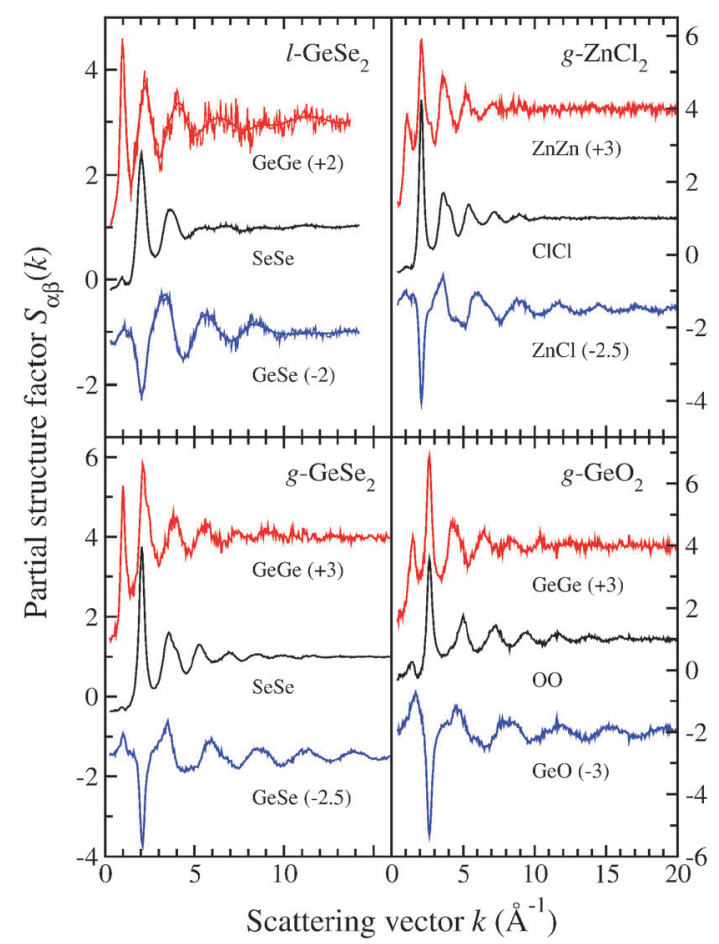

Fig. 2 The measured Faber-Ziman partial structure factors $S_{\alpha \beta}(k)$ for liquid $\mathrm{GeSe}_{2},{ }^{66,67}$ and for the glasses $\mathrm{GeSe}_{2}{ }^{67,68} \mathrm{ZnCl}_{2}$ (ref. 53 and 69) and $\mathrm{GeO}_{2}{ }^{70,71}$ In each panel, the upper (red) curve gives $S_{\mathrm{MM}}(k)$, the middle (black) curve gives $S_{X X}(k)$, and the lower (blue) curve gives $S_{M X}(k)$. The statistical uncertainties are represented by the scatter in the data points. In the case of liquid $\mathrm{GeSe}_{2}$, the ratio of the liquid to melting-point temperatures, $T / T_{\mathrm{mp}}$ is 1.041 . The diffraction measurements for the glasses were all made at $\simeq 298 \mathrm{~K}$. Figure from Salmon. ${ }^{72}$ (C) IOP Publishing. Reproduced with permission. All rights reserved. 


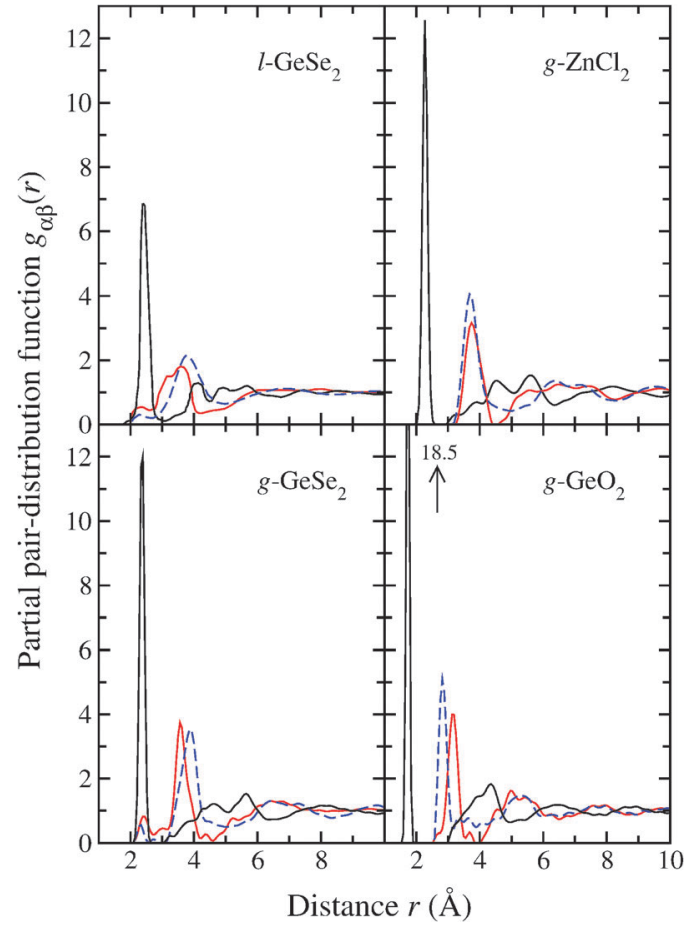

Fig. 3 The measured partial pair-distribution functions $g_{\alpha \beta}(r)$ for liquid $\mathrm{GeSe}_{2},{ }^{66,67}$ and for the glasses $\mathrm{GeSe}_{2},{ }^{67,68} \mathrm{ZnCl}_{2}$ (ref. 53 and 69) and $\mathrm{GeO}_{2} \cdot{ }^{70,71}$ In each panel, the dark (black) curve gives $g_{\mathrm{MX}}(r)$, the light (red) curve gives $g_{\mathrm{MM}}(r)$ and the broken (blue) curve gives $g_{x x}(r)$. In the case of the glasses, the effect in real space of truncating the diffraction pattern at a finite maximum value $k_{\max }$ before Fourier transformation was taken into account by using the procedure described by Salmon and Petri. ${ }^{67}$ The oscillations at $r$-values smaller than the distance of closest approach between two atoms have been suppressed for clarity of presentation. Figure from Salmon. ${ }^{72}$ (C) IOP Publishing. Reproduced with permission. All rights reserved.

in $\mathrm{GeO}_{2}$ glass, while edge-sharing tetrahedra also appear in $\mathrm{ZnCl}_{2}$ and $\mathrm{GeSe}_{2}$, their numbers being greater for the liquid by comparison with the glass. The appearance of these edgesharing conformations is consistent with the more fragile characters of $\mathrm{ZnCl}_{2}$ and $\mathrm{GeSe}_{2}$ by comparison with $\mathrm{GeO}_{2} \cdot{ }^{75}$ Homopolar Ge-Ge and Se-Se bonds are also an important feature in liquid and glassy $\mathrm{GeSe}_{2}$. In the case of the glass, the first three peaks in $g_{\text {GeGe }}(r)$ correspond with increasing $r$ to the $\mathrm{Ge}-\mathrm{Ge}$ distances in homopolar bonds, edge-sharing tetrahedra and corner-sharing tetrahedra, respectively. ${ }^{67,68}$

The $\mathrm{X}$-atom packing fraction $\eta_{\mathrm{X}}$, as calculated by assuming spherical $\mathrm{X}$ atoms arranged in $\mathrm{MX}_{4}$ tetrahedra, is smaller for $\mathrm{GeO}_{2}$ by comparison with $\mathrm{ZnCl}_{2}$, in keeping with a larger intertetrahedral $\mathrm{M}-\mathrm{X}-\mathrm{M}$ bond angle, and in the case of $\mathrm{ZnCl}_{2}$ glass ${ }^{53,81}$ its magnitude is close to the value of $\simeq 0.64$ expected for a random close-packing of hard spheres. ${ }^{82}$ The high values of $\eta_{\mathrm{X}}$ for liquid and glassy $\mathrm{GeSe}_{2}$ could be attributed to the relative softness of Se atoms, although the presence of nontetrahedral motifs such as homopolar bonds will lead to smaller calculated $\eta_{\mathrm{X}}$ values. ${ }^{72}$

For $\mathrm{MX}_{2}$ materials, the Bhatia-Thornton pair-correlation functions have proved to be an instructive way of decomposing the structure into its contributions from the topological and chemical ordering. ${ }^{45,72}$ The measured Bhatia-Thornton partial structure factors $S_{\mathrm{IJ}}(k)$ for liquid and glassy $\mathrm{GeSe}_{2}$, glassy $\mathrm{ZnCl}_{2}$ and glassy $\mathrm{GeO}_{2}$ are illustrated in Fig. 4. The FSDP and principal peak appear, to a greater or lesser extent, as features in all of the measured $S_{\mathrm{IJ}}(k)$ functions and, for a given material, the FSDP positions are comparable as are the principal peak positions. The principal peak is the dominant feature in $S_{\mathrm{CC}}(k)$ and corresponds to chemical ordering (i.e. a preference for $\mathrm{M}-\mathrm{X}$ nearest-neighbours) in real space. In comparison, $S_{\mathrm{NN}}(k)$ is more structured with a ratio of FSDP to principal peak heights that correlates with the system's fragility. For example, the FSDP in $S_{\mathrm{NN}}(k)$ is a comparatively large feature for the strong glass-forming material $\mathrm{GeO}_{2}$ but is a comparatively small feature for the more fragile glass-forming material $\mathrm{ZnCl}_{2}$. Hence, there is an interplay between the relative importance of the length scales associated with the intermediate versus extended ranges and, with increasing fragility, it is the extended-range ordering which dominates. ${ }^{70} \|$

The character of the observed extended-range order can be investigated by considering a simple rigid-ion pair-potential model for the interactions between two particles labelled by $i$ and $j$ separated by a distance $r$ as represented by the expression $^{83}$

$$
\phi_{i j}(r)=\phi_{i j}^{\mathrm{sr}}(r)+\frac{Z_{i} Z_{j} e^{2}}{\varepsilon r}-\frac{A_{i j}}{r^{6}}
$$

where $Z_{i} e$ is the charge on the $i$ th ion, $e$ is the elementary charge, $\varepsilon \equiv 4 \pi \varepsilon_{r} \varepsilon_{0}, \varepsilon_{r}$ is the dimensionless relative dielectric constant of the medium in which the ions are embedded, and $\varepsilon_{0}$ is the vacuum permittivity. In eqn (14), $\phi_{i j}^{\mathrm{sr}}(r)$ is a shortranged repulsive term, $\phi_{i j}^{\text {Coul }}(r) \propto r^{-1}$ is a Coulomb term, and $\phi_{i j}^{\text {disp }}(r)=-A_{i j} r^{-6}$ is a dispersion term in which $A_{i j}$ is a parameter $(\geq 0)$ that depends on the polarisability of the ions. ${ }^{84}$

For the rigid-ion model, a simple power-law dependence for the ultimate decay of the pair correlation functions is expected i.e. $r h_{\mathrm{NN}}(r) \rightarrow r^{-5}, r h_{\mathrm{NC}}(r) \rightarrow r^{-7}, r h_{\mathrm{CC}}(r) \rightarrow r^{-9}$ where $h_{\mathrm{NN}}(r) \equiv$ $g_{\mathrm{NN}}(r)-1, h_{\mathrm{NC}}(r) \equiv g_{\mathrm{NC}}(r), h_{\mathrm{CC}}(r) \equiv g_{\mathrm{CC}}(r) .{ }^{40,85}$ However, if the dispersion terms are absent in eqn (14), then a pole analysis of the $k$-space solutions to the Ornstein-Zernike equations following the method of Evans and co-workers ${ }^{38,39}$ leads, in the case when the system density is high, to the following expressions for the asymptotic decay of the pair-correlation functions $^{40}$

$$
\begin{gathered}
r h_{\mathrm{NN}}(r) \rightarrow 2\left|\mathscr{A}_{\mathrm{NN}}\right| \exp \left(-a_{0} r\right) \cos \left(a_{1} r-\theta_{\mathrm{NN}}\right) \\
r h_{\mathrm{CC}}(r) \rightarrow 2 c_{\mathrm{M}} c_{\mathrm{X}}\left|\mathscr{A}_{\mathrm{CC}}\right| \exp \left(-a_{0} r\right) \cos \left(a_{1} r-\theta_{\mathrm{CC}}\right) \\
r h_{\mathrm{NC}}(r) \rightarrow 2\left|\mathscr{A}_{\mathrm{NC}}\right| \exp \left(-a_{0} r\right) \cos \left(a_{1} r-\theta_{\mathrm{NC}}\right)
\end{gathered}
$$

|| The appearance of ordering on both the intermediate and extended length scales is sometimes unappreciated for prototypical network glass-forming systems such as $\mathrm{SiO}_{2}$ and $\mathrm{GeO}_{2}$ because the principal peak is usually small or absent in the measured neutron or X-ray diffraction patterns. However, as shown by the measured partial structure factors for $\mathrm{SiO}_{2}$ and $\mathrm{GeO}_{2}$ glass, ${ }^{71,74}$ the suppression of this feature simply reflects the fact that the weighting factors in eqn (2) lead to a cancellation of the principal peaks in $S_{\mathrm{MM}}(k)$ and $S_{\mathrm{XX}}(k)$ with the principal trough in $S_{\mathrm{Mx}}(k)$. 
Table 1 The position of the main peak $r_{\alpha \beta}$ and corresponding coordination number $\bar{n}_{\alpha}^{\beta}$ for the $g_{\alpha \beta}(r)$ functions measured for several liquid and glassy network-forming materials with the $\mathrm{MX}_{2}$ stoichiometry. In the case of liquid and glassy $\mathrm{GeSe}_{2}$, peaks also occur in $g_{\mathrm{GeGe}}(r)$ and $g_{\text {sese }}(r)$ at smaller $r$-values. The ratio of the X-X distance $r_{X X}$ to the $\mathrm{M}-\mathrm{X}$ distance $r_{\mathrm{MM}}$ is also given where, for regular tetrahedral $\mathrm{MX}$ units, $r_{\mathrm{XX}} / r_{\mathrm{MX}}=\sqrt{8 / 3}=1.633$. The $\mathrm{M}-\mathrm{X}-\mathrm{M}$ bond angle $\theta_{\mathrm{MXM}}$ is calculated from $r_{\mathrm{MX}}$ and the $\mathrm{M}-\mathrm{M}$ distance $r_{\mathrm{MM}}$ by using $\cos \left(\theta_{\mathrm{MXM}}\right)=1-r_{\mathrm{MM}}{ }^{2} / 2 r_{\mathrm{MX}}{ }^{2}$. For an $\mathrm{MX}$ system, the X-atom number density $\rho_{\mathrm{X}}=2 \rho / 3$ such that, if these atoms are assumed to be hard spheres of radius $r_{X}$, then their packing fraction $\eta_{X}=(8 / 9) \rho \pi r_{X}^{3}$. If the X-atoms are also touching in regular $\mathrm{MX}_{4}$ tetrahedra then $r_{\mathrm{XX}}=2 r_{\mathrm{X}}$ such that the packing fraction can be re-written as $\eta_{\mathrm{X}}=16 \sqrt{2} \pi \rho r_{\mathrm{MX}}{ }^{3} / 27 \sqrt{3}=1.520 \rho r_{\mathrm{MX}}{ }^{3}$

\begin{tabular}{|c|c|c|c|c|c|c|c|}
\hline System & $g_{\alpha \beta}(r)$ & $r_{\alpha \beta}(\AA)$ & $\bar{n}_{\alpha}^{\beta}$ & $r_{\mathrm{XX}} / r_{\mathrm{MX}}$ & $\mathrm{M}-\mathrm{X}-\mathrm{M}\left({ }^{\circ}\right)$ & $\eta_{\mathrm{X}}$ & Ref. \\
\hline l-GeSe ${ }_{2}$ & $\begin{array}{l}\mathrm{GeSe} \\
\mathrm{GeGe}^{a} \\
\mathrm{SeSe}^{a}\end{array}$ & $\begin{array}{l}2.42(2) \\
3.59(2) \\
3.75(2)\end{array}$ & $\begin{array}{l}3.5(2) \\
2.9(3) \\
9.6(3)\end{array}$ & $1.550(15)$ & $96(1)$ & $0.670(17)$ & 66,67 \\
\hline $\mathrm{g}-\mathrm{GeSe}_{2}$ & $\begin{array}{l}\mathrm{GeSe} \\
\mathrm{GeGe}^{b} \\
\mathrm{SeSe}^{b}\end{array}$ & $\begin{array}{l}2.36(2) \\
3.57(2) \\
3.89(2)\end{array}$ & $\begin{array}{l}3.7(1) \\
3.2(3) \\
9.3(2)\end{array}$ & $1.648(16)$ & $80(1)^{c}, 98(1)$ & $0.667(17)$ & 67,68 \\
\hline $1-\mathrm{ZnCl}_{2}$ & $\begin{array}{l}\mathrm{ZnCl} \\
\mathrm{ZnZn} \\
\mathrm{ClCl}\end{array}$ & $\begin{array}{l}2.27(2) \\
3.96(3), 3.74(5)^{d} \\
3.68(3)\end{array}$ & $\begin{array}{l}4.2(2) \\
3.8(2), 4.20(1)^{d} \\
10.0(2)\end{array}$ & $1.621(19)$ & $121(2), 111(3)^{d}$ & $0.596(16)$ & 53 \\
\hline $\mathrm{g}-\mathrm{ZnCl}_{2}$ & $\begin{array}{l}\mathrm{ZnCl} \\
\mathrm{ZnZn} \\
\mathrm{ClCl}\end{array}$ & $\begin{array}{l}2.28(1) \\
3.75(1) \\
3.70(1)\end{array}$ & $\begin{array}{r}3.9(1) \\
4.0(1) \\
12.1(2)\end{array}$ & $1.623(8)$ & $111(1)$ & $0.647(9)$ & 53,69 \\
\hline $\mathrm{g}-\mathrm{GeO}_{2}$ & $\begin{array}{l}\mathrm{GeO} \\
\text { GeGe } \\
\mathrm{OO}\end{array}$ & $\begin{array}{l}1.73(1) \\
3.16(1) \\
2.83(1)\end{array}$ & $\begin{array}{l}3.8(1) \\
4.1(2) \\
6.7(1)\end{array}$ & $1.636(11)$ & $132(2)$ & $0.495(9)$ & 70,71 \\
\hline
\end{tabular}

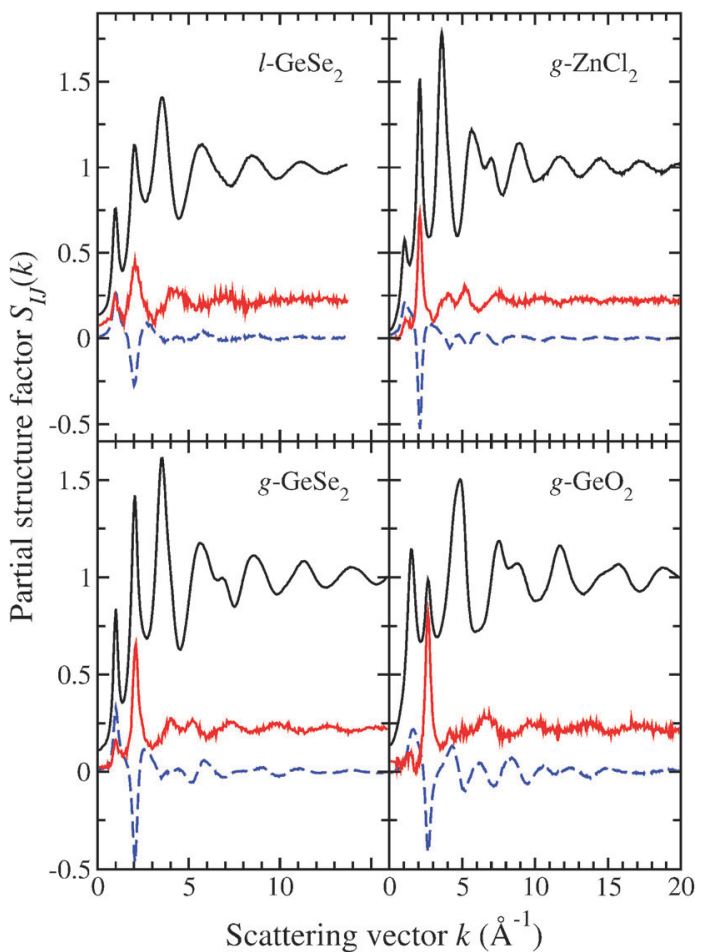

Fig. 4 The measured Bhatia-Thornton partial structure factors $S_{1 J}(k)$ for liquid $\mathrm{GeSe}_{2},{ }^{66,67}$ and for the glasses $\mathrm{GeSe}_{2},{ }^{67,68} \mathrm{ZnCl}_{2}$ (ref. 53 and 69) and $\mathrm{GeO}_{2}{ }^{70,71}$ In each panel, the upper (black) curve gives $S_{\mathrm{NN}}(k)$, the middle (red) curve gives $S_{C C}(k)$ and the lower (blue) broken curve gives $S_{N C}(k)$. The statistical uncertainties are represented by the scatter in the data points. Figure from Salmon. ${ }^{72}$ (C) IOP Publishing. Reproduced with permission. All rights reserved.
The $r h_{\mathrm{IJ}}(r)$ are therefore exponentially damped oscillatory functions with a common decay length given by $a_{0}{ }^{-1}$ and a common wavelength for the oscillations given by $2 \pi / a_{1} \cdot{ }^{* *}$ The $\mathscr{A}_{\mathrm{IJ}}$ are complex numbers with amplitudes related by $\left|\mathscr{A}_{\mathrm{NN}}\right|\left|\mathscr{A}_{\mathrm{CC}}\right|=$ $\left|\mathscr{A}_{\mathrm{NC}}\right|^{2}$ and phases related by $\theta_{\mathrm{NN}}+\theta_{\mathrm{CC}}=2 \theta_{\mathrm{NC}}$. Eqn (15)-(17) also hold for hard-sphere systems when both the Coulomb and dispersion terms are absent from eqn (14). Thus, although they are derived from a simple theory, eqn (15)-(17) provide a convenient starting point for investigating the extended-range order in liquid and glassy materials.

To illustrate the ordering on an extended length scale in $\mathrm{MX}_{2}$ network glass-forming materials, it is instructive to consider the results for glassy $\mathrm{ZnCl}_{2}$ (ref. 69) since it is a system that is expected to fall within the framework of an ionic interaction model, provided that ion polarisation effects are taken into account. ${ }^{24}$ The measured real-space functions $r h_{\mathrm{IJ}}(r)$ and $\ln \left|r h_{\mathrm{IJ}}(r)\right|$ are plotted in Fig. 5 and show ordering at large- $r$ values which persists to distances far exceeding the correlation length $2 \pi / \Delta k_{\mathrm{FSDP}} \simeq$ 12.6 $\AA$ estimated from the width of the FSDP in $S_{\mathrm{NN}}(k)$. The extended range oscillations in glassy $\mathrm{ZnCl}_{2}$ show exponential decay with a common inverse decay length $a_{0} \simeq 0.19 \AA^{-1}$ and a periodicity that is governed not by the position of the FSDP but by the position of the principal peak, giving oscillations of

** For low densities, the $r h_{\mathrm{IJ}}(r)$ functions show simple exponential decay with a common decay length. The crossover from pure exponential to exponentially damped oscillatory decay defines a line in the density-temperature plane known either as the Fisher-Widom line or as the Kirkwood line, depending on the mechanism by which crossover occurs. ${ }^{38-40}$ 


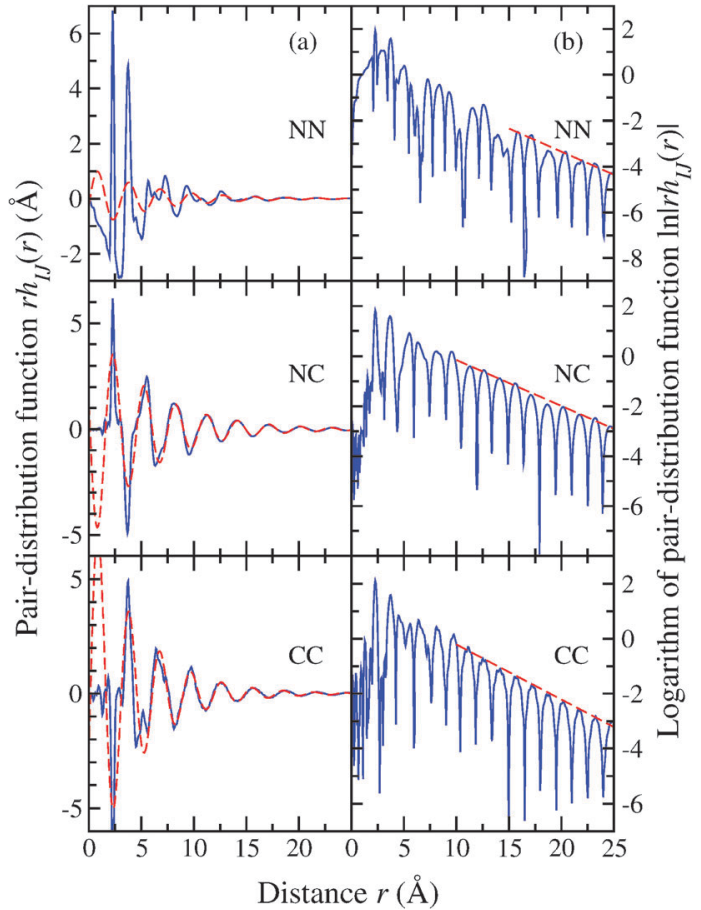

Fig. 5 Decay of the Bhatia-Thornton partial pair-distribution functions for glassy $\mathrm{ZnCl}_{2}$ as shown by plotting in column (a) $r h_{\mathrm{J}}(r)$ versus $r$ (solid (blue) curves) and in column (b) $\ln \left|r h_{\mathrm{IJ}}(r)\right|$ versus $r$ (solid (blue) curves). ${ }^{40}$ In column (a), the broken (red) curves show fits to the $r h_{\mathrm{J}}(r)$ functions at large $r$-values using eqn (15)-(17). In column (b), the broken (red) curves show fits of the repeated maxima at large- $r$ to a straight line $\left(\ln \left|r h_{1 \mathrm{~J}}(r)\right|=-a_{0} r+\right.$ constant) from which the decay length $a_{0}{ }^{-1}$ can also be found.

wavelength $2 \pi / a_{1} \simeq 2 \pi / k_{\mathrm{PP}}$. Indeed, eqn (16) and (17) provide a robust account of the measured $r h_{\mathrm{CC}}(r)$ and $r h_{\mathrm{NC}}(r)$ functions from large- $r$ to distances as short as $\simeq 5 \AA$. The relationship between the amplitudes predicted by simple theory does not, however, appear to hold. Similar conclusions are drawn from an analysis of the measured $r h_{\mathrm{IJ}}(r)$ functions for $\mathrm{GeSe}_{2}$ and $\mathrm{GeO}_{2}$ glass. ${ }^{72} \dagger \uparrow$ The presence of extended-range ordering in network glass-forming systems has been confirmed by molecular dynamics simulations using a polarisable ion model to describe the atomic interactions. ${ }^{89}$

For the investigated $\mathrm{MX}_{2}$ network glass-forming materials, the wavelength of the extended range oscillations is comparable to the size of a tetrahedral $\mathrm{MX}_{4}$ motif i.e. $2 \pi / k_{\mathrm{PP}} \simeq 4 r_{\mathrm{MX}} / 3$ where the latter is the base-to-apex distance in a regular tetrahedron and $r_{\mathrm{MX}}$ is the nearest-neighbour $\mathrm{M}-\mathrm{X}$ distance. ${ }^{72}$ Although homopolar bonds appear in materials like $\mathrm{GeSe}_{2}$, there is a preference for chemical ordering as manifested by the large principal peak in $S_{\mathrm{CC}}(k)$. The tetrahedra therefore pack to give number-density fluctuations, as represented by oscillations at large- $r$ in $r h_{\mathrm{NN}}(r)$, that occur on a very similar length

†† Exponentially damped oscillatory $r h_{\mathrm{IJ}}(r)$ functions for network glasses will not be reproduced by models based on crystalline systems since the ordering in $r h_{\mathrm{IJ}}(r)$ will persist to arbitrarily large distances, ${ }^{86}$ even when a large positional disorder is applied to the atomic coordinates. ${ }^{87,88}$ scale to the concentration fluctuations, as represented by oscillations at large- $r$ in $r h_{\mathrm{CC}}(r)$. The structural motifs also organise on an intermediate length scale via the formation of ring structures, thus providing a modulation of the pairdistribution functions on this length scale that manifests itself by the appearance of an FSDP in reciprocal space.

\subsection{Specific issues regarding the structure of $\mathrm{GeSe}_{2}$}

The validity of the NDIS results for liquid and glassy $\mathrm{GeSe}_{2}$ has been called into question by some authors.

Petkov and Le Messurier ${ }^{90}$ measured a single X-ray total structure factor $S_{\mathrm{X}}(k)$ and constructed an RMC model in which the glass structure is described in terms of a continuous network of corner- and edge-sharing $\mathrm{GeSe}_{4}$ tetrahedra with no homopolar bonds. As discussed in ref. 90, the differences with the NDIS results presented in Section 3.1 could originate, in part, from the use of different sample preparation methods. However, the atomic numbers of Ge and Se are similar such that their atomic form factors are close to one another. From eqn (7) and (11) it follows that, in a conventional X-ray diffraction experiment, the weighting factors for $S_{\mathrm{NN}}(k), S_{\mathrm{CC}}(k)$ and $S_{\mathrm{NC}}(k)$ in the expression for $S_{\mathrm{X}}(k)$ are e.g. 1, 0.0036 and -0.12 at $k=0$ such that $S_{\mathrm{X}}(k) \simeq S_{\mathrm{NN}}(k)$. Hence, $S_{\mathrm{X}}(k)$ will contain negligible information on the function $S_{\mathrm{CC}}(k)$ which describes the chemical ordering i.e. the $\mathrm{X}$-rays cannot distinguish between Ge and Se atoms (Section 2.1). In comparison, the observation by the NDIS method of homopolar bonds in $\mathrm{GeSe}_{2}$ glass is consistent with the findings from Raman, Mössbauer and X-ray emission spectroscopy experiments, ${ }^{67,91,92}$ and the concentration of homopolar bonds found from the NDIS experiments is in agreement with an estimate based on the law of mass action. $^{67,68}$

Soper ${ }^{93}$ has discussed the relatively weak contribution of Ge-Ge correlations to the measured diffraction patterns for $\mathrm{GeSe}_{2}$ glass, using the EPSR method with simple reference pairpotentials护 to model the measured total structure factors. As expected from an SVD analysis, ${ }^{53}$ the Ge-Ge correlations were found to be most sensitive to the details of the model, and doubt was cast on the reality of structural features such as $\mathrm{Ge}-\mathrm{Ge}$ homopolar bonds. In comparison, the $\mathrm{GeSe}_{2}$ system has been the subject of extensive first-principles molecular dynamics simulations in which the electronic structure of the material is taken into explicit account, as befits an ionocovalent bonded system in which the electronegativity of the different chemical species is similar. ${ }^{29,95-110}$ In the work of Drabold and co-workers, features in the NDIS results such as homopolar Ge-Ge and Se-Se bonds and the appearance of an FSDP in $S_{\mathrm{CC}}(k)$, were reproduced in the first-principles models. ${ }^{95,96,100}$ In the work of Massobrio and co-workers, it initially proved difficult to reproduce these features which led inter alia to the use of different functionals for the electron

护 The reference pair-potentials used to produce EPSR models usually comprise a Lennard-Jones term, a Coulomb term to represent charged atom interactions, and often an additional repulsive exponential term aimed at softening the LennardJones repulsive core. ${ }^{33,93,94}$ 
exchange and correlation..$^{97-99,101,103,105} \S \S$ Nowadays, use of the Becke, Lee, Yang, and Parr (BLYP) functional, which enhances a localised distribution of the valence electrons, is leading to much improved agreement with the NDIS results. ${ }^{29,107-110}$ These developments have also been accompanied by improvements in the simulated diffusion coefficients of the melt. ${ }^{107,109}$ Thus, the partial structure factors obtained directly from the measured diffraction data, ${ }^{66-68}$ as opposed to modelling those data, ${ }^{93}$ appear to provide the most realistic picture for the structure of liquid and glassy $\mathrm{GeSe}_{2}$. $\uparrow$ All of this is helping to provide a framework for e.g. interpreting ${ }^{73} \mathrm{Ge}$ and ${ }^{77} \mathrm{Se}$ solid state nuclear magnetic resonance spectra for Ge-Se glasses, investigations that also find evidence for broken chemical order in $\mathrm{GeSe}_{2} \cdot{ }^{111-114}$

\subsection{Concentration fluctuations on an intermediate length scale}

The observation of an FSDP in $S_{\mathrm{CC}}(k)$ for several $\mathrm{MX}_{2}$ network glass-forming materials (Fig. 4$)^{45,66}$ has been a source of controversy because it was not predicted from the investigation of these materials by using classical molecular dynamics simulations or integral-equation calculations. ${ }^{115-118}$ Also, if these materials can be treated as purely ionic, i.e. as a two-component system of pointlike cations and anions, then the charge-charge partial structure factor is given by $S_{\mathrm{ZZ}}(k)=S_{\mathrm{CC}}(k) / c_{\mathrm{M}} c_{\mathrm{X}}$ such that an FSDP in $S_{\mathrm{CC}}(k)$ implies a non-uniformity in the charge distribution on an intermediate length scale. ${ }^{45}$

Extensive first-principles molecular dynamics investigations, in which $S_{\mathrm{CC}}(k)$ is calculated from the atomic positions and $S_{\mathrm{zZ}}(k)$ is calculated by taking into account the valence-electron density, show that these fluctuations in charge do not occur. ${ }^{29,102}$ Instead, the absence of charge fluctuations over intermediate range distances provides a constraint on the network properties. ${ }^{102}$ For example, in chemically ordered systems like $\mathrm{SiO}_{2}$, where the valence state of a given chemical species is invariant, the net charge on a network-forming structural motif is zero so there is no need for concentration fluctuations to occur on an intermediate range. In contrast, for systems like $\mathrm{GeSe}_{2}$ and $\mathrm{SiSe}_{2}$, there is a moderate number of chemical defects which leads to a local variation in the charge on network-forming structural motifs. These motifs must then link to satisfy charge neutrality on the length scale of a few structural motifs, leading to the appearance of concentration fluctuations on this intermediate length scale. In the case of liquid $\mathrm{GeSe}_{2}$, a significant

$\S \S$ The work in ref. 95, 96 and 100 used an electronic-structure scheme within the local density approximation (LDA) for the exchange and correlation energy which does not evolve self-consistently with the atomic motion, together with a minimal basis set. Several of the features in the resultant models are consistent with experiment $^{67}$ but are not reproduced when using fully self-consistent LDA calculations. This is attributed, in the latter, to an underestimate in the ionic character of the bonding. ${ }^{97-99,101}$

IT When using the EPSR method to extract partial structure factors, it is possible to weight the results either towards those obtained from the reference rigid-ion pair-potential model, such that the extracted pair-correlation functions are essentially those of this starting model, or towards the experimental data, such that the extracted pair-correlation functions contain more information from the experimental results. ${ }^{33}$ It is therefore possible to bias the results towards those expected from the starting model. contribution to the FSDP in $S_{\mathrm{CC}}(k)$ is likely to arise from short chains of edge-sharing tetrahedra in which the Ge atoms at opposite chain ends are separated from each other by intermediate range distances and where the overall unit has the valence state of a mis-coordinated Ge atom. ${ }^{29,104}$ Eventually, as the degree of chemical disorder increases and the network structure breaks-up, the FSDP disappears from the total structure factor and vanishes from $S_{\mathrm{CC}}(k)$. Thus, the concentrationconcentration partial structure factor has proved to be a sensitive probe of the chemical bonding in $\mathrm{MX}_{2}$ systems.

\section{Liquid and amorphous metals: extended-range ordering}

The measured structure factors of metallic liquids and glasses are usually dominated by a first sharp peak in the range $k \simeq$ 2.2-2.9 $\AA^{-1},{ }^{123,124}$ which will be referred to as the principal peak in keeping with the description of binary network glass-forming materials given in Section 3. This is illustrated by the measured neutron total structure factors $S_{\mathrm{N}}(k)$ shown in Fig. 6 for the liquid alloys $\mathrm{Au}_{0.81} \mathrm{Si}_{0.19}, \mathrm{Au}_{0.72} \mathrm{Ge}_{0.28}$ and $\mathrm{Ag}_{0.74} \mathrm{Ge}_{0.26}$ which have compositions at or near to the eutectic. \|\| The fragility of glass-forming liquid metals can take a wide range of values, ${ }^{127,128}$ there being a trend towards less fragile behaviour with increasing number of chemical components. ${ }^{128}$ The materials generally have densely-packed structures, ${ }^{129}$ and so-called bulk metallic alloys made by using low cooling-rates have applications as engineering materials. ${ }^{128,130-133}$ The nature of the structural motifs, their organisation at distances larger than the nearestneighbour distance, and the relationship between structure and issues such as glass-forming ability, remain the subjects of debate. ${ }^{129,134-136 * * *}$ As in Section 3, we associate extendedrange ordering with the principal peak, although the structure in disordered metals at distances beyond the nearest-neighbour is usually called medium-range ordering in the literature on these materials. ${ }^{124,129,134,136}$

The asymptotic decay of the measured total pair-distribution functions for a variety of metallic glasses has been interpreted by Ma et $a l .^{124}$ in terms of a self-similar packing of atomic clusters which leads to a fractal network of dimension $D_{\mathrm{f}}$. Specifically, if it is assumed that the clusters show a degree of self-similarity or scale invariance, and that the principal peak position in $S_{\mathrm{X}}(k)$ or $S_{\mathrm{N}}(k)$ is related to the atomic volume $v$ as calculated from the measured density by using $k_{\mathrm{PP}} \propto v^{-1 / D_{\mathrm{f}}}$, then it follows that ${ }^{119,124}$

$$
\ln k_{\mathrm{PP}}=\ln a-\ln v / D_{\mathrm{f}}
$$

|| || The formation of metallic glasses was first reported for the $\mathrm{Au}-\mathrm{Si}$ system near the eutectic composition. ${ }^{125,126}$

*** In principle, liquid and glassy metals should be treated as a mixture of ions and valence electrons such that the structure, for example, of a single component system is described in terms of ion-ion $S_{\mathrm{II}}(k)$, ion-valence electron $S_{\mathrm{Ie}}(k)$, and valence electron-valence electron $S_{\mathrm{ee}}(k)$ partial structure factors. ${ }^{137}$ Since neutrons scatter from nuclei whereas X-rays scatter from electrons, it is possible in some materials like liquid $\mathrm{Li}$ to extract $S_{\mathrm{II}}(k)$ and $S_{\mathrm{Ie}}(k)$ along with the valence electron form factor. ${ }^{138-141}$ 


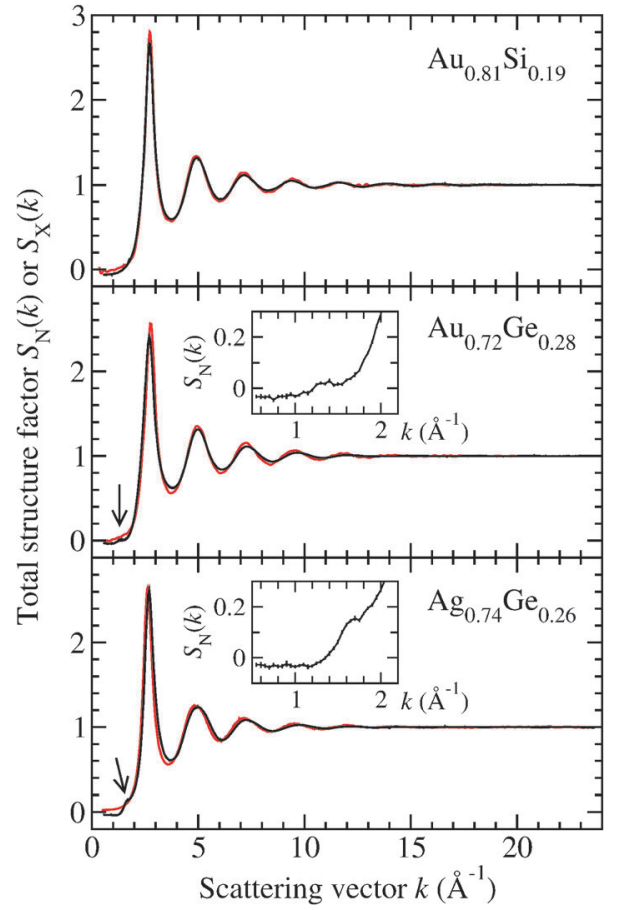

Fig. 6 The total neutron structure factors $S_{N}(k)$ as measured for liquid $\mathrm{Au}_{0.81} \mathrm{Si}_{0.19}$ at $665 \mathrm{~K}, \mathrm{Au}_{0.72} \mathrm{Ge}_{0.28}$ at $666 \mathrm{~K}$, and $\mathrm{Ag}_{0.74} \mathrm{Ge}_{0.26}$ at $976 \mathrm{~K}$ (solid dark (black) curves). The line thickness is greater than the size of the vertical error bars at most $k$ values. ${ }^{119}$ The arrows point to small prepeaks in $S_{N}(k)$ at $\simeq 1.3$ and $1.6 \AA^{-1}$ for the Au-Ge and Ag-Ge alloys, respectively, and the insets show an expanded view of the low- $k$ region for $S_{N}(k)$. The total structure factors $S_{X}(k)$ as measured for the liquid alloys at similar temperatures by using high-energy $\mathrm{X}$-ray diffraction are also shown (solid light (red) curves). ${ }^{120-122}$

where $a$ is a constant of proportionality. As shown by Fig. 7(a), the data for a wide variety of glassy metals can be fitted using eqn (18) which leads to $D_{\mathrm{f}}=2.31$. The asymptotic decay of a measured total pair-distribution function was then fitted by Ma et al. ${ }^{124}$ using

$$
r^{D^{\prime}}\left[G_{\mathrm{N}}(r)-1\right] \simeq A \exp \left(-a_{0} r\right) \cos \left(a_{1} r-\phi\right)
$$

where $D^{\prime} \equiv D-D_{\mathrm{f}}=0.69$ ( $D=3$ is the embedding dimension), $A$ is an amplitude and $\phi$ is a phase. $+\dagger$ The oscillatory term, of wavelength $2 \pi / a_{1}$, was included in order to modify an expression previously used to model the small-angle neutron scattering data measured for silica particle aggregates, where $a_{0}{ }^{-1}$ was a cut-off length chosen to ensure that the aggregates (or clusters) were not infinite in extent. ${ }^{142}$ In the fitting procedure, $a_{1}$ was set equal to the principal peak position $k_{\mathrm{Pp} .}{ }^{124}$

It is, however, possible to account for the extended-range ordering associated with the principal peak in liquid and glassy metals without invoking fractal dimensionality. ${ }^{119}$ For example, the available data for metallic glasses can be equally well fitted by using the relation $k_{\mathrm{PP}} \propto v^{-1 / 3}$ as expected for a threedimensional structure (Fig. 7(b)). Also, the extended-range ordering can be accounted for by making a pole analysis of the

†† Eqn (19) was assumed by Ma et al. ${ }^{124}$ to be valid for both neutron and X-ray diffraction data.
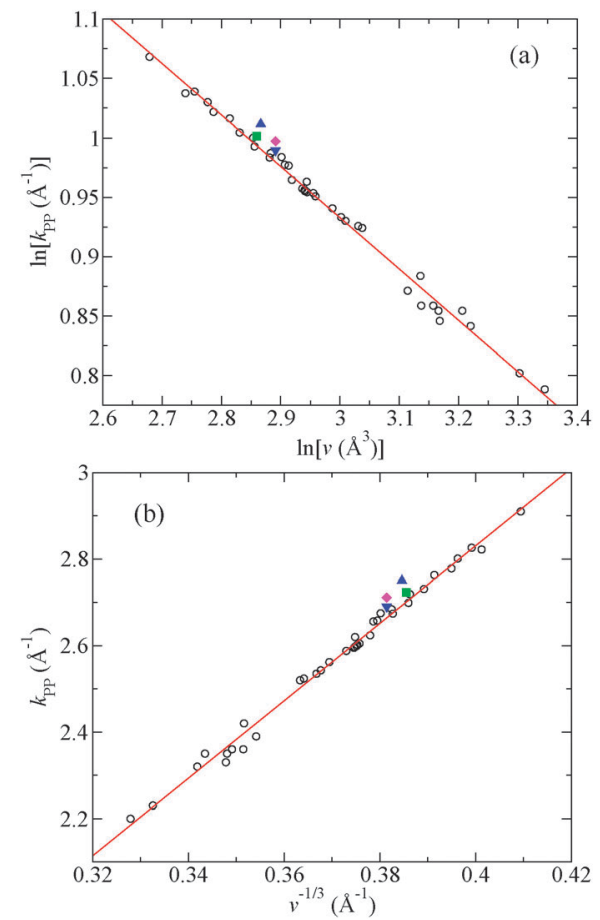

Fig. 7 Plots of (a) $\ln k_{\mathrm{PP}} v s . \ln v$ and (b) $k_{\mathrm{PP}} v s . v^{-1 / 3}$ for a selection of metallic glasses and liquids where $k_{\mathrm{pp}}$ is the position of the principal peak in a measured total structure factor and $v$ is the atomic volume. ${ }^{119}$ The open circles show the data points for various glasses taken from Ma et al. ${ }^{124}$ and the solid (red) curves show straight-line fits. Also shown are the data points for glassy $\mathrm{Au}_{0.80} \mathrm{Si}_{0.20}$ ((blue) $\boldsymbol{\Delta})$ and for liquid $\mathrm{Au}_{0.81} \mathrm{Si}_{0.19}$ at $665 \mathrm{~K}$ ((green) $\left.\boldsymbol{\square}\right), \mathrm{Au}_{0.72} \mathrm{Ge}_{0.28}$ at $666 \mathrm{~K}$ ((magenta) $\bullet$ ) and $\mathrm{Ag}_{0.74} \mathrm{Ge}_{0.26}$ at $976 \mathrm{~K}$ ((blue) $\left.\mathbf{\nabla}\right)$. $^{19,143}$ Reprinted with permission from Chirawatkul et al. ${ }^{119}$ Copyright (2011) by the American Physical Society.

Ornstein-Zernike equations, an approach that has been used to describe the asymptotic behaviour of the pair-distribution functions for hard-sphere mixtures. ${ }^{144,145}$ If the dispersion term is absent from eqn (14) then, irrespective of whether the Coulomb term is absent or present, the asymptotic behaviour of a partial pair-distribution function for sufficiently high particle density is described by the relation ${ }^{38,39}$

$$
r\left[g_{\alpha \beta}(r)-1\right]=2\left|\mathscr{A}_{\alpha \beta}\right| \exp \left(-a_{0} r\right) \cos \left(a_{1} r-\theta_{\alpha \beta}\right)
$$

where each function has a common decay length given by $a_{0}{ }^{-1}$ and a common wavelength of oscillation given by $2 \pi / a_{1}$. The amplitudes are related by $\left|\mathscr{A}_{\alpha \alpha}\right|\left|\mathscr{A}_{\beta \beta}\right|=\left|\mathscr{A}_{\alpha \beta}\right|^{2}$ and the phases are related by $\theta_{\alpha \alpha}+\theta_{\beta \beta}=2 \theta_{\alpha \beta}$. By using the definition of the total pair-distribution function given by eqn (12) it follows that ${ }^{119}$

$$
r\left[G_{\mathrm{N}}(r)-1\right]=2|\mathscr{A}| \exp \left(-a_{0} r\right) \cos \left(a_{1} r-\theta\right)
$$

where $\mathscr{A}=\left(c_{\alpha} b_{\alpha} \mathscr{A}_{\alpha \alpha}{ }^{1 / 2}+c_{\beta} b_{\beta} \mathscr{A}_{\beta \beta}{ }^{1 / 2}\right)^{2} /\langle b\rangle^{2} \equiv|\mathscr{A}| \exp (-i \theta), \mathscr{A}_{\alpha \alpha}=$ $\left|\mathscr{A}_{\alpha \alpha}\right| \exp \left(-i \theta_{\alpha \alpha}\right), \mathscr{A}_{\beta \beta}=\left|\mathscr{A}_{\beta \beta}\right| \exp \left(-i \theta_{\beta \beta}\right), i=\sqrt{-1}$ and $\theta$ is a phase.㧊 The only significant difference between eqn (19)

持 It is necessary to assume that the weighting factors for the $r\left[g_{\alpha \beta}(r)-1\right]$ terms are independent of $r$ if eqn (21) is also to apply to X-ray diffraction data. In general these weighting factors are, however, related to Fourier transforms of the atomic form factors $f(k)$ and will therefore be $r$-dependent. ${ }^{119}$ 


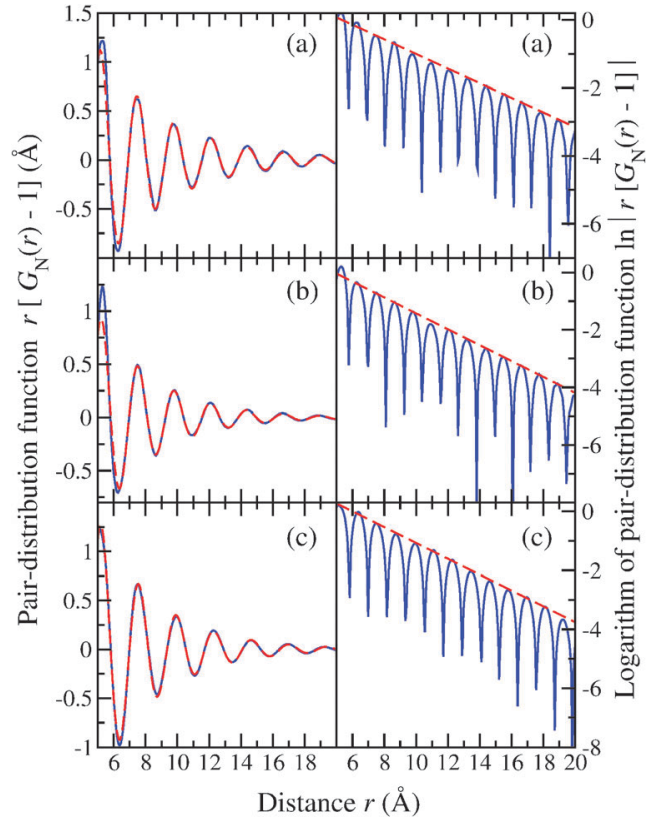

Fig. 8 Decay of the total pair-distribution functions for liquid (a) $A u_{0.81} S_{0} i_{019}$ at $665 \mathrm{~K}$, (b) $\mathrm{Au}_{0.72} \mathrm{Ge}_{0.28}$ at $666 \mathrm{~K}$, and (c) $\mathrm{Ag}_{0.74} \mathrm{Ge}_{0.26}$ at $976 \mathrm{~K}$. In the left-hand column, the measured functions $r\left[G_{N}(r)-1\right]$ (solid dark (blue) curves) are fitted to eqn (21) at large-r, and for a given liquid alloy the values of $a_{0}$ and $a_{1}$ obtained from the fits are comparable to the half-width at half-maximum of the principal peak in $S_{N}(k)$ and to the position $k_{\mathrm{PP}}$ of this peak, respectively. In the right-hand column, the solid dark (blue) curves give $\ln \left|r\left[G_{N}(r)-1\right]\right|$ and the broken (red) curves show fits to the repeated maxima at large- $r$ to a straight line $\left(\ln \left|r\left[G_{N}(r)-1\right]\right|=-a_{0} r+\right.$ constant $)$ from which the decay length $a_{0}{ }^{-1}$ can also be found. Reprinted with permission from Chirawatkul et al. ${ }^{119}$ Copyright (2011) by the American Physical Society.

and (21) is the power of $r$ used to weight $G_{\mathrm{N}}(r)-1$ and, as illustrated in Fig. 8, eqn (21) can be used to account for the measured behaviour of the total pair-distribution functions for several liquid eutectic alloys over a large $r$-range. A similar conclusion is anticipated for the glassy metals studied by Ma et al. ${ }^{124}$ given the similarity between the equations obtained from the fractal and Ornstein-Zernike approaches. The fractal approach for describing extended-range ordering in liquid and glassy metals is not, therefore, convincing.

\section{Liquids at high-temperatures: vitrification of fragile glass-forming systems}

Containerless high-temperature levitation methods offer several attractions for investigating liquids. ${ }^{146}$ For example, contamination of the liquid by reaction with container walls is avoided and crystal formation by heterogeneous nucleation is minimised, allowing liquids to be deeply supercooled. This can extend the number of glass-forming systems and/or the range of glass-forming compositions such that it is possible to make new glasses with novel physical properties. ${ }^{147,148}$ A disadvantage for the NDIS method is that the samples are small, being roughly spherical with a diameter of $\sim 8 \mathrm{~mm}$ for electromagnetic-levitation experiments ${ }^{149}$ or $2-4 \mathrm{~mm}$ for aerodynamic-levitation experiments. ${ }^{150,151} \S \S \S$ Nevertheless, the NDIS method with electromagnetic levitation has been applied to measure the partial structure factors of liquid $\mathrm{Ni}_{36} \mathrm{Zr}_{64}{ }^{152,153}$ Its isomorphic substitution counterpart has also been combined with electromagnetic levitation to measure the partial structure factors of stable and supercooled liquid $\mathrm{Al}_{13}\left(\mathrm{Co}_{x} \mathrm{Fe}_{1-x}\right)_{4}(0 \leq x \leq 1)$, where isomorphism was assumed for the transition metals Co and Fe. ${ }^{154}$ ศा 9 It is only recently, however, that the NDIS method with aerodynamic-levitation and laser-heating has been used to investigate the structure of oxides, where initial investigations have focussed on the fragile glass-forming systems $\mathrm{CaAl}_{2} \mathrm{O}_{4}$ (see Fig. 1$)^{18}$ and $\mathrm{CaSiO}_{3} .{ }^{19}$

Consider the case of $\mathrm{CaAl}_{2} \mathrm{O}_{4}$, and let diffraction patterns be measured for three samples that are identical in every respect, except for the isotopic composition of calcium which is of natural isotopic abundance ${ }^{\text {nat }} \mathrm{Ca}$, predominantly the isotope ${ }^{44} \mathrm{Ca}$, or a 50:50 mixture of the two ${ }^{\text {mix }} \mathrm{Ca}$. Let the corresponding scattering lengths be denoted by $b_{\text {nat }} \mathrm{Ca}, b_{44} \mathrm{Ca}$ and $b_{\text {mix }}$ and the measured total structure factors be represented by ${ }^{\text {nat }} F(k),{ }^{44} F(k)$ and ${ }^{\mathrm{mix}} F(k)$, respectively. ${ }^{18}$ In matrix notation,

$$
\left[\begin{array}{l}
{ }^{\text {nat }} F(k) \\
{ }^{44} F(k) \\
{ }^{\text {mix }} F(k)
\end{array}\right]=\left[\begin{array}{lll}
c_{\mathrm{Ca}^{2}}{ }^{2} b_{\text {nat }}{ }^{2} & 2 c_{\mathrm{Ca}} b_{\text {nat }} \mathrm{Ca} & 1 \\
c_{\mathrm{Ca}}{ }^{2} b_{44} \mathrm{Ca}^{2} & 2 c_{\mathrm{Ca}} b_{44} \mathrm{Ca} & 1 \\
c_{\mathrm{Ca}}{ }^{2} b_{\text {mix }}{ }^{2} & 2 c_{\mathrm{Ca}} b_{\text {mix }} & 1
\end{array}\right]\left[\begin{array}{l}
S_{\mathrm{CaCa}}(k)-1 \\
\Delta F_{\mathrm{Ca}}(k) \\
\Delta F_{\mu \mu}(k)
\end{array}\right]
$$

where

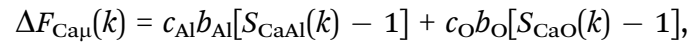

$\mu$ denotes $\mathrm{Al}$ or $\mathrm{O}$, and

$$
\begin{aligned}
\Delta F_{\mu \mu}(k)= & c_{\mathrm{Al}}^{2} b_{\mathrm{Al}}^{2}\left[S_{\mathrm{AlAl}}(k)-1\right]+c_{\mathrm{O}}{ }^{2} b_{\mathrm{O}}{ }^{2}\left[S_{\mathrm{OO}}(k)-1\right] \\
& +2 c_{\mathrm{Al}} c_{\mathrm{O}} b_{\mathrm{Al}} b_{\mathrm{O}}\left[S_{\mathrm{AlO}}(k)-1\right] .
\end{aligned}
$$

The corresponding real-space functions $\Delta G_{\mathrm{Ca \mu}}(r)$ and $\Delta G_{\mu \mu}(r)$ are obtained by substituting $S_{\alpha \beta}(k)$ for $\mathrm{g}_{\alpha \beta}(r)$ in eqn (23) and (24), respectively. Hence, it is possible to separate the measured diffraction patterns into their contributions from the $\mathrm{Ca}-\mathrm{Ca}$, $\mathrm{Ca}-\mu$ and $\mu-\mu$ correlations.

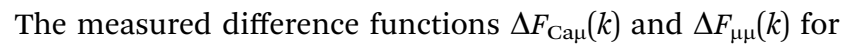
the liquid at 1973(30) $\mathrm{K}$ and for the glass at 300(1) $\mathrm{K}$ are shown in Fig. 9 and the corresponding real-space functions $\Delta G_{\text {Cau }}(r)$ and $\Delta G_{\mu \mu}(r)$ are shown in Fig. 10. In the case of the glass, the sample was not levitated but was rested on the nozzle of the levitation device, and improved counting statistics made it possible to extract the Ca-Ca partial structure factor $S_{\mathrm{CaCa}}(k)$ and corresponding partial pair-correlation function $d_{\mathrm{CaCa}}(r) \equiv$ $4 \pi \rho r\left[g_{\mathrm{CaCa}}(r)-1\right]$ which are shown in Fig. 11.

The results were interpreted with the aid of molecular dynamics simulations, using interaction potentials that take

$\S \S \S$ In the case of aerodynamic-levitation experiments, a large fraction of the sample volume is shielded from an incident neutron or X-ray beam by the nozzle of the levitation device. ${ }^{150}$

ฯबा In the isomorphic substitution method, different elements with contrasting scattering lengths are substituted for one another. It is assumed that these elements have identical coordination environments. 


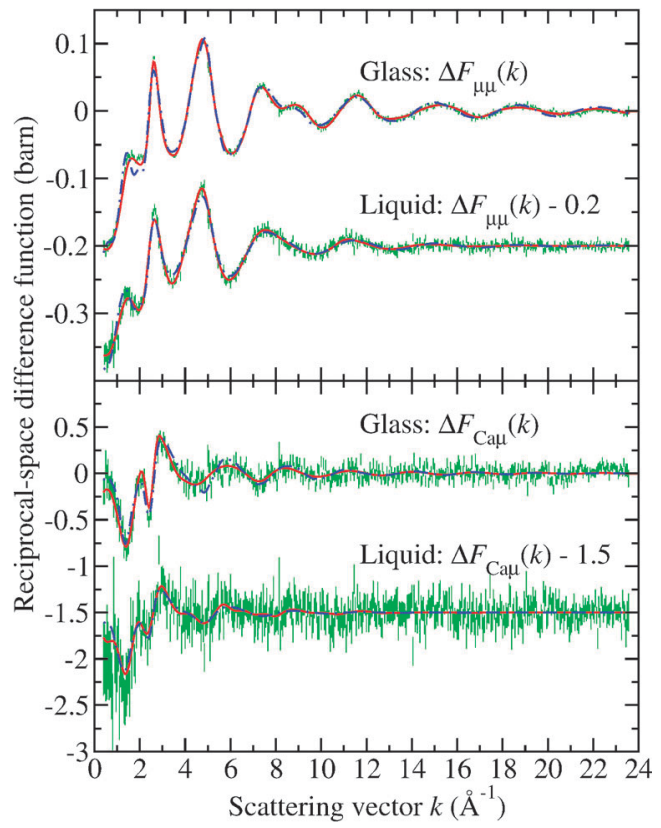

Fig. 9 The difference functions $\Delta F_{\mu \mu}(k)$ and $\Delta F_{\text {Сa }}(k)$ for glassy and liquid $\mathrm{CaAl}_{2} \mathrm{O}_{4} \cdot{ }^{18}$ The vertical bars show the statistical errors on the measured data points, and the solid (red) curves show the back Fourier transforms of the corresponding real-space functions shown by the solid (black) curves in Fig. 10. The chained (blue) curves show the molecular dynamics results obtained by using a polarisable-ion model in which anion polarisability and shape-deformation effects are taken into account along with the polarisability of the calcium cations..$^{27,155}$

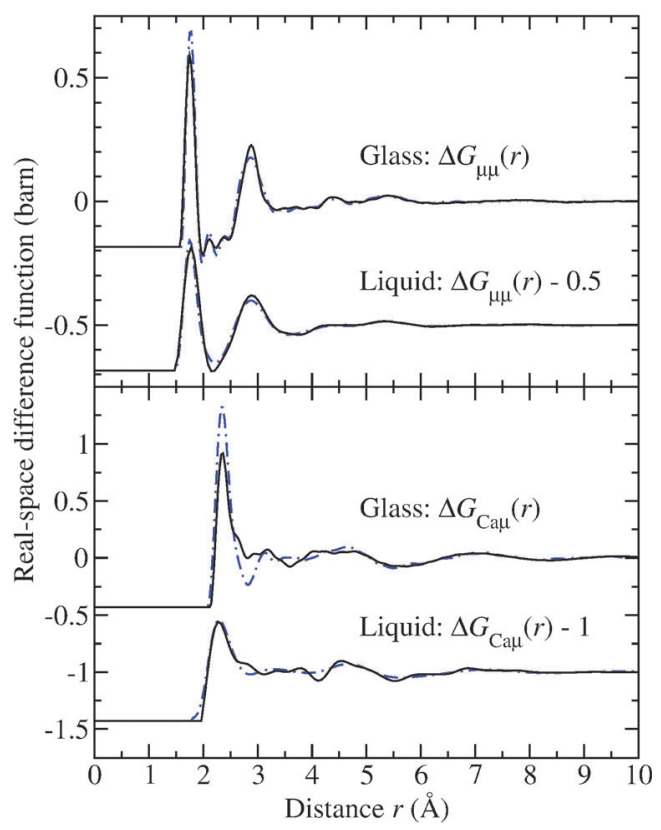

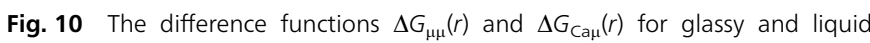
$\mathrm{CaAl}_{2} \mathrm{O}_{4}$ as obtained by Fourier transforming either the spline-fitted measured (solid (black) curves) or the simulated (chained (blue) curves) reciprocal-space functions shown in Fig. 9. The unphysical small-r oscillations below the distances of closest interatomic approach are omitted for clarity of presentation.

into account anion polarisability and shape-deformation effects as well as the polarisability of the calcium cations, ${ }^{27,155}$ and revealed
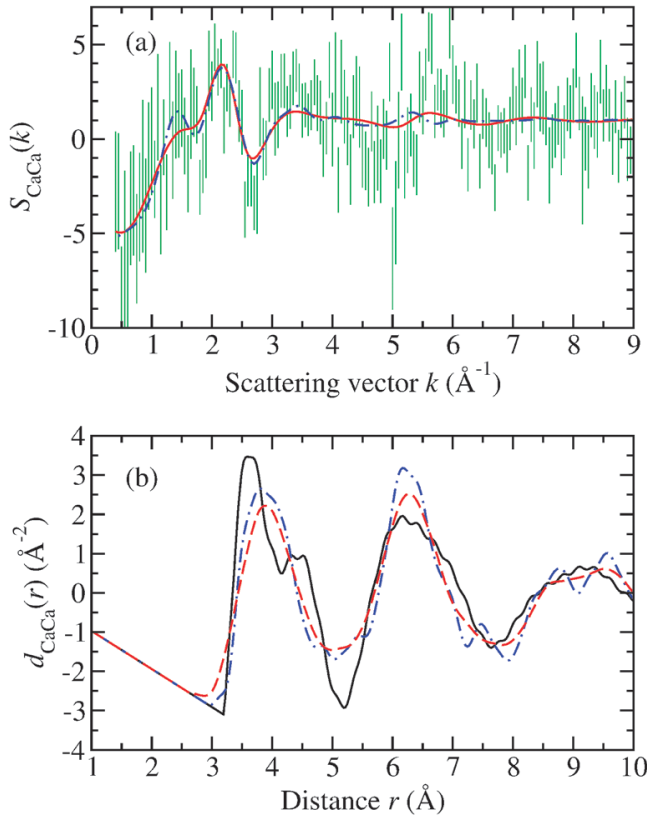

Fig. 11 (a) The measured (points with vertical error bars) and simulated (chained (blue) curve) partial structure factors $\mathrm{S}_{\mathrm{CaCa}}(k)$ for $\mathrm{CaAl}_{2} \mathrm{O}_{4}$ glass. ${ }^{18}$ The solid (red) curve shows the back Fourier transform of $d_{\mathrm{CaCa}}(r)$ given in (b) by the solid (black) curve. (b) The partial pair-correlation function $d_{\mathrm{CaCa}}(r)$ as obtained by Fourier transforming the spline-fitted measured (solid (black) curve) or the simulated (broken (red) curve) $S_{C_{a} c_{a}}(k)$ function shown in (a) after applying a Lorch modification function with $k_{\max }=8.5 \AA^{-1}$. The chained (blue) curve shows the results obtained directly from the molecular dynamics simulation (see the text). Reprinted with permission from Drewitt et al. ${ }^{18}$ Copyright (2012) by the American Physical Society.

key structural modifications upon glass formation on multiple length scales. Specifically, there is a reorganisation on quenching that leads to an almost complete breakdown of the $\mathrm{AlO}_{5}$ polyhedra and threefold coordinated oxygen atoms present in the liquid, and to their replacement by a predominantly corner-sharing network of $\mathrm{AlO}_{4}$ tetrahedra with twofold coordinated oxygen atoms in the glass. This process is accompanied by the formation of branched-chains of edge- and face-sharing Ca-centered polyhedra that give cationic ordering on an intermediate length scale, where the measured Ca-O coordination numbers are $\bar{n}_{\mathrm{Ca}}^{\mathrm{O}}=6.0(2)$ for the liquid and $\bar{n}_{\mathrm{Ca}}^{\mathrm{O}}=6.4$ (2) for the glass. \|\|$\|$ The formation of more extensively linked chains of Ca-centred polyhedra on vitrification is also indicated by the joint NDIS and molecular dynamics investigation of $\mathrm{CaSiO}_{3}$ by Skinner et al. ${ }^{19}$

Since glasses can explore localities on an energy landscape that are inaccessible to the crystalline state, they are widely used

|| || | These Ca-O coordination numbers were obtained by integrating over the first peak in the relevant $\Delta G_{\text {Cap }}(r)$ function up to a cut-off value $r_{\text {cut }}=3 \AA$ (eqn (4)). For the glass, a reduced coordination number of $\bar{n}_{\mathrm{Ca}}^{\mathrm{O}}=5.1(2)$ is obtained by shortening $r_{\text {cut }}$ to $2.79 \AA$, in accord with new results obtained from work using density functional theory and reverse Monte Carlo modelling. ${ }^{156}$ In the latter work, the fraction of oxygen atoms in the glass that form triclusters, where one oxygen atom is shared by three $\mathrm{AlO}_{4}$ tetrahedra, was found to be $\sim 14.5 \%$ from the models, somewhat larger than the fraction of $\sim 5 \%$ found from nuclear magnetic resonance experiments ${ }^{157}$ and the estimate of $2(2) \%$ from the NDIS work. ${ }^{18}$ 
as proxies for the liquid in materials of geophysical interest. ${ }^{158-163}$ Although this is known to be an approximation, ${ }^{162,163}$ the extreme experimental conditions make it difficult to assess the extent of associated structural differences. The results for liquid and glassy $\mathrm{CaAl}_{2} \mathrm{O}_{4}$ show significant structural re-organization on vitrification in this fragile glass former, a taxonomy that encompasses a range of magma-related liquids. ${ }^{164-166}$ There is a change not only in the character of the network-forming motifs but also in the nature of the intermediate-ranged cationic ordering. The work therefore cautions against the use of glasses as proxies for investigating the structure of fragile glass-forming liquids that are of geophysical interest.

The NDIS method, or its isomorphic substitution counterpart, has previously been used to investigate the structure of a wide variety of metal-containing multi-component liquids and glasses, allowing a measured total structure factor to be separated either into its contributions from the $\mathbf{M}-\mathbf{M}, \mathbf{M}-\mu$ and $\mu-\mu$ pair-correlations, where $\mathbf{M}$ denotes a metal atom and $\mu$ denotes the other chemical species, ${ }^{64,167-171}$ or into difference functions that otherwise simplify the complexity of correlations. ${ }^{172-180}$ The results obtained by Drewitt et al. ${ }^{18}$ demonstrate that it is now feasible to use this methodology to study the detailed structure of small oxide samples, including single liquid droplets under high-temperature conditions.

\section{Glasses at high-pressures: mechanisms of density-driven network collapse}

The structural changes in glasses and liquids induced by highpressure and/or high-temperature conditions can alter substantially their dynamical and transport properties. ${ }^{6,37,181,182}$ A notable example is provided by so-called polyamorphic transitions, where the variation of pressure and/or temperature leads to an abrupt transformation between two phases having identical compositions but different densities. ${ }^{6,37,151,181-184}$ The nature of structural disorder, combined with the experimental difficulties associated with the investigation of materials under extreme conditions, ${ }^{60,185-188}$ provide severe challenges to unravelling the mechanisms by which these structural transformations occur. Recently, however, Wezka et al. ${ }^{20}$ have made the first application of the NDIS method to measure the structure of $\mathrm{GeO}_{2}$ glass with a Paris-Edinburgh press at pressures ranging from ambient to $\sim 8 \mathrm{GPa}$. Valuable new information was thereby provided on the mechanisms of density-driven network collapse.

Let diffraction experiments be made on samples of ${ }^{70} \mathrm{GeO}_{2}$ and ${ }^{73} \mathrm{GeO}_{2}$ glass that are identical in every respect, except for their Ge isotope enrichments, such that the total structure factors ${ }^{70} F(k)$ and ${ }^{73} F(k)$ are measured, respectively. Then the contribution to the diffraction patterns from the $\mathrm{O}-\mathrm{O}$ partial structure factor can be eliminated by forming the first-difference function

$$
\begin{aligned}
\Delta F_{\mathrm{Ge}}(k) \equiv & { }^{70} F(k)-{ }^{73} F(k) \\
= & 2 c_{\mathrm{Ge}} c_{\mathrm{O}} b_{\mathrm{O}}\left(b_{70} \mathrm{Ge}-b_{73} \mathrm{Ge}\right. \\
& +c_{\mathrm{Ge}}{ }^{2}\left(b_{70}{ }_{\mathrm{Ge}}{ }^{2}-b_{73}{ }_{\mathrm{GeO}}^{2}\right)\left[S_{\mathrm{GeGe}}(k)-1\right] .
\end{aligned}
$$

Alternatively, the contribution to the diffraction patterns from the Ge-Ge partial structure factor can be eliminated by forming the weighted first-difference function

$$
\begin{aligned}
& \Delta F_{\mathrm{O}}(k) \equiv\left[b_{73} \mathrm{Ge}{ }^{270} F(k)-b_{70} \mathrm{Ge}^{273} F(k)\right] /\left(b_{73} \mathrm{Ge}^{2}-b_{70} \mathrm{Ge}^{2}\right) \\
& =2 c_{\mathrm{Ge}} c_{\mathrm{O}} b_{\mathrm{O}} b_{70}{ }_{\mathrm{Ge}} b_{73 \mathrm{Ge}}\left(b_{70} \mathrm{Ge}+b_{73} \mathrm{Ge}\right)^{-1} \\
& \times\left[S_{\mathrm{GeO}}(k)-1\right]+c_{\mathrm{O}}^{2} b_{\mathrm{O}}^{2}\left[S_{\mathrm{OO}}(k)-1\right] .
\end{aligned}
$$

The corresponding real-space functions $\Delta G_{\mathrm{Ge}}(r)$ and $\Delta G_{\mathrm{O}}(r)$ are obtained by substituting $S_{\alpha \beta}(k)$ for $g_{\alpha \beta}(r)$ in eqn (25) and (26), respectively.

The pressure dependence of the measured ${ }^{70} F(k)$ and ${ }^{73} F(k)$ functions is shown in Fig. 12, where ${ }^{73} F(k)$ is dominated by the Bhatia-Thornton number-number partial structure factor $S_{\mathrm{NN}}(k)$ because the scattering lengths of oxygen and the isotope ${ }^{73} \mathrm{Ge}$ are similar (see eqn (7)). With increasing density, the position of the FSDP moves to higher $k$ values as the principal peak sharpens. These changes indicate a competition between the intermediate and extended-range ordering which is won by the latter with increasing density, consistent with the transformation from a "strong" low-density to a more "fragile" high-density glass (see Section 3.1) ${ }^{70,72}$ Similar behaviour is observed in high-pressure X-ray diffraction experiments on $\mathrm{GeSe}_{2}$ glass, ${ }^{191}$ where $S_{\mathrm{X}}(k) \simeq$ $S_{\mathrm{NN}}(k)$ because $f_{\mathrm{Ge}}(k) \simeq f_{\mathrm{Se}}(k)$ (Section 3.2).****

The pressure dependence of the measured difference functions $\Delta F_{\mathrm{Ge}}(k)$ and $\Delta F_{\mathrm{O}}(k)$ for $\mathrm{GeO}_{2}$ glass is shown in Fig. 13. The robustness of the results was thoroughly checked by using the procedures described in ref. 20. The corresponding real-space

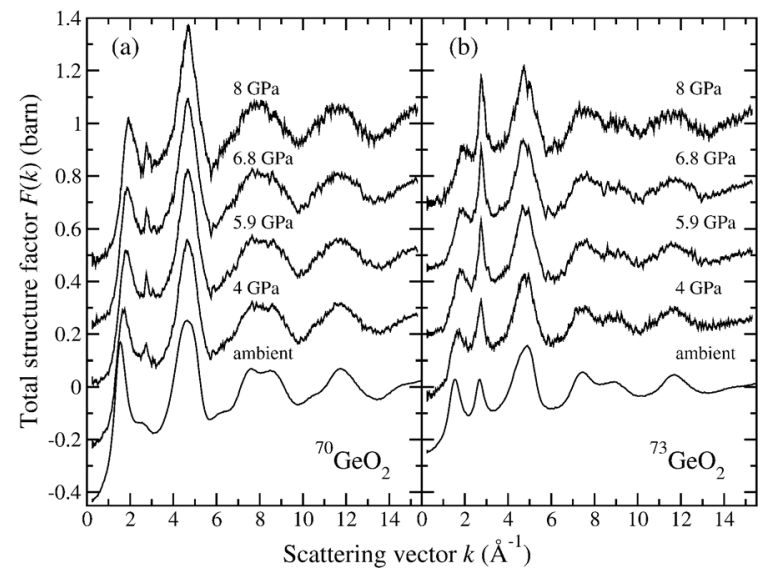

Fig. 12 The pressure dependence of the measured total structure factors (a) ${ }^{70} F(k)$ and $(b){ }^{73} F(k)$ for $\mathrm{GeO}_{2}$ glass. ${ }^{20}$ According to eqn (11), these functions can be re-written as $S_{N}(k) \equiv F(k) /\langle b\rangle^{2}+1$ in which the weighting factors for the Bhatia-Thornton $S_{N N}(k), S_{C C}(k)$ and $S_{N C}(k)$ partial structure factors are (a) 1, 0.3344 and 1.1565 or (b) $1,0.0137$ and -0.2338 , respectively, i.e. ${ }^{73} F(k)$ is dominated by $S_{N N}(k)$. Vertical bars give the statistical errors on the measured data points, and the high-pressure data sets have been displaced vertically for clarity of presentation.

\footnotetext{
**** The principal peak in the measured neutron diffraction pattern for liquid ammonia also sharpens with increasing density as packing constraints become more important. ${ }^{192}$ The extended-range ordering for this system is described by using eqn (21).
} 


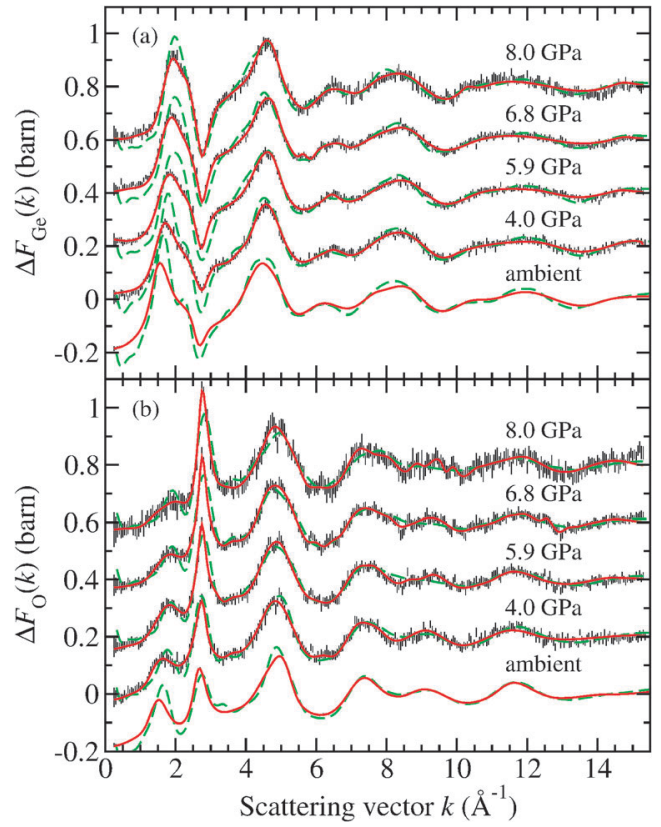

Fig. 13 The pressure dependence of the difference functions (a) $\Delta F_{\mathrm{Ge}}(k) / \mathrm{barn}=$ $0.124(3)\left[S_{\mathrm{GeO}}(k)-1\right]+0.081(2)\left[S_{\mathrm{GeGe}}(k)-1\right]$ and $(\mathrm{b}) \Delta F_{\mathrm{O}}(k) / \mathrm{barn}=$ $0.0875(5)\left[S_{G e O}(k)-1\right]+0.1497(2)\left[S_{O O}(k)-1\right]$ for $\mathrm{GeO}_{2}$ glass. ${ }^{20}$ The vertical bars give the statistical errors on the measured data points, the solid (red) curves give the Fourier transforms of the corresponding real-space functions shown in Fig. 14, and the broken (green) curves give the molecular dynamics results obtained by using transferrable interaction potentials that include dipole-polarisation effects. ${ }^{28,36}$ The high-pressure data sets have been shifted vertically for clarity of presentation. Figure from Wezka et al. ${ }^{20}$ (C) IOP Publishing. Reproduced with permission. All rights reserved.

functions $\Delta G_{\mathrm{Ge}}(r)$ and $\Delta G_{\mathrm{O}}(r)$ are illustrated in Fig. 14 where the first peak, which is attributable to Ge-O correlations, gives the Ge-O bond distances and coordination numbers shown in Fig. 15. Importantly, the NDIS method allows for the nearestneighbour $\mathrm{Ge}-\mathrm{Ge}$ and $\mathrm{O}-\mathrm{O}$ correlations to be resolved, as manifested by the second peaks in $\Delta G_{\mathrm{Ge}}(r)$ and $\Delta G_{\mathrm{O}}(r)$, respectively. The corresponding distances are plotted in Fig. 15 along with the $\mathrm{O}-\mathrm{O}$ coordination number $\bar{n}_{\mathrm{O}}^{\mathrm{O}}$, obtained by assuming minimal overlap with the Ge-O correlations as observed under ambient conditions. ${ }^{71}$ In Fig. $13-15$, the diffraction results are compared to molecular dynamics simulations made using interaction potentials that include dipole-polarisation effects. ${ }^{20,28,36}$ The potentials were parameterised using $a b$ initio simulations as opposed to experimental results and are therefore largely unbiased in their predictions of the glass structure. In contrast to other models for $\mathrm{GeO}_{2}$ glass under pressure, ${ }^{193-197}$ the experimental and simulation results are consistent with one another, an agreement that also extends to the pressure dependence of the mean $\mathrm{O}-\mathrm{Ge}-\mathrm{O}$ and $\mathrm{Ge}-\mathrm{O}-\mathrm{Ge}$ bond angles. ${ }^{20}$

It is concluded that when the reduced density $\rho / \rho_{0}<1.16$, where $\rho_{0}$ is the value at ambient pressure, compaction proceeds via a reorganisation of distorted corner-sharing $\mathrm{GeO}_{4}$ tetrahedra in which there is a reduction of the mean $\mathrm{Ge}-\mathrm{O}-\mathrm{Ge}$ bond angle and an increase in $\bar{n}_{\mathrm{O}}^{\mathrm{O}}$. On further densification, $\mathrm{GeO}_{4}$ units are replaced mostly by distorted square pyramidal $\mathrm{GeO}_{5}$ units,

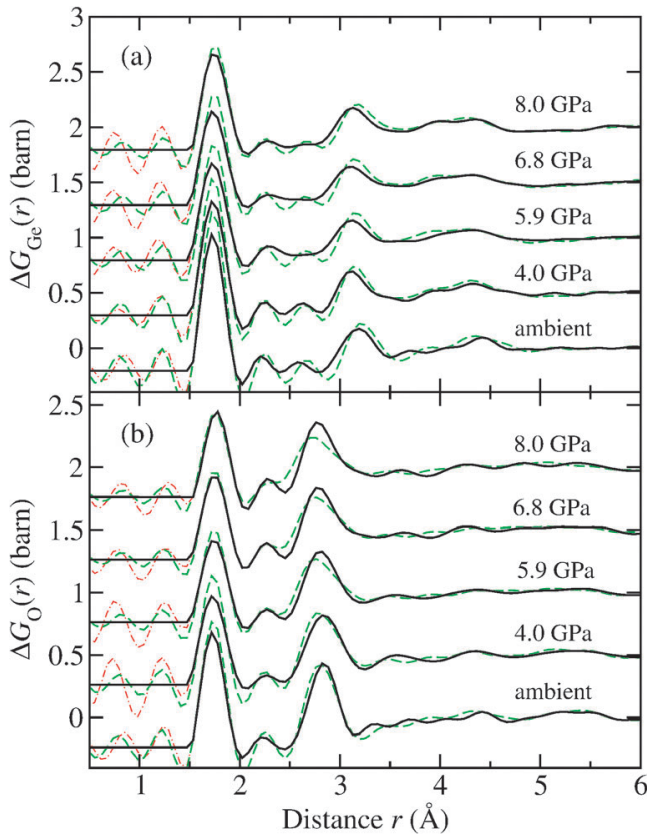

Fig. 14 The pressure dependence of the difference functions (a) $\Delta G_{G e}(r) /$ barn $=$ $0.124(3)\left[g_{\mathrm{GeO}}(r)-1\right]+0.081(2)\left[g_{\mathrm{GeGe}}(r)-1\right]$ and (b) $\Delta G_{\mathrm{O}}(r) / \mathrm{barn}=$ $0.0875(5)\left[g_{\text {Geo }}(r)-1\right]+0.1497(2)\left[g_{\mathrm{oo}}(r)-1\right]$ (solid (black) curves), as obtained by spline fitting and Fourier transforming the measured reciprocal-space functions shown in Fig. 13, where the chained (red) curves show the oscillations at $r$-values smaller than the distance of closest approach between two atoms. The broken (green) curves give the molecular dynamics results as obtained by Fourier transforming the simulated functions shown in Fig. 13 after applying the same maximum cutoff $k_{\max }$ as for the neutron diffraction data. The high-pressure data sets have been shifted vertically for clarity of presentation. Figure from Wezka et al. ${ }^{20}$ (C) IOP Publishing. Reproduced with permission. All rights reserved.

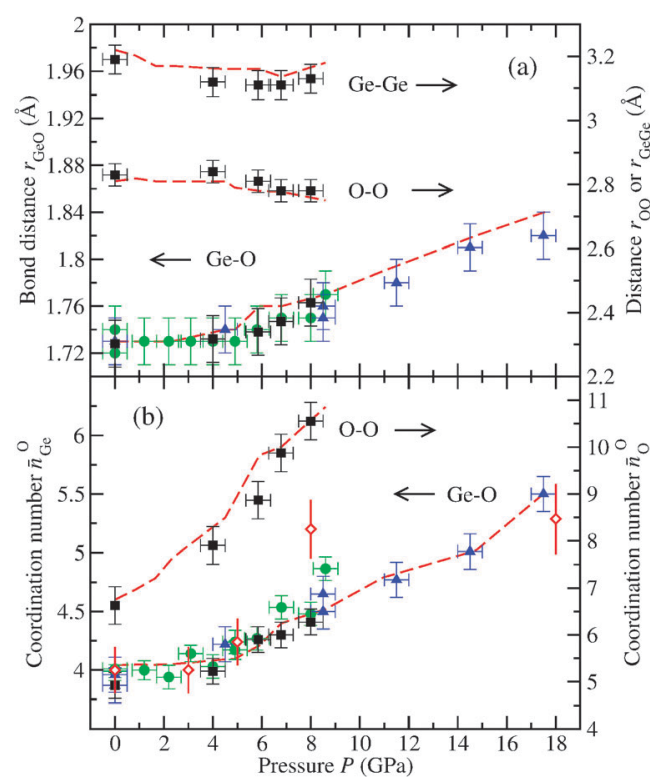

Fig. 15 The pressure dependence of the nearest-neighbour (a) Ge-O, O-O and $\mathrm{Ge}-\mathrm{Ge}$ distances and (b) Ge-O and O-O coordination numbers. The results from the neutron diffraction ( $\mathbf{\square}$ ) and molecular dynamics (broken (red) curves) work of Wezka et al..$^{20}$ are compared to those obtained from the neutron diffraction studies of Drewitt et $a l^{60}$ ((green) $\bullet$ ) and Salmon et al. ${ }^{61}$ ((blue) $\left.\mathbf{\Delta}\right)$. In (b) the Ge-O coordination numbers from inelastic $X$-ray scattering experiments ${ }^{189}$ are also shown $(($ red $) \diamond)$. Figure from Wezka et al. ${ }^{20}$ C) IOP Publishing. Reproduced with permission. All rights reserved. 
where the vacancy at the base of the latter anticipates the eventual formation of octahedral (i.e. square bipyramidal) units. At higher pressures, $\mathrm{GeO}_{6}$ units begin to form when $\rho / \rho_{0} \gtrsim 1.4$ and the fraction of $\mathrm{GeO}_{5}$ units reaches a maximum when $\rho / \rho_{0}=1.45$. Thus when $\rho / \rho_{0} \gtrsim 1.16$, the replacement of tetrahedral $\mathrm{GeO}_{4}$ units proceeds via an interplay between the predominance of distorted square pyramidal $\mathrm{GeO}_{5}$ units versus octahedral $\mathrm{GeO}_{6}$ units. $^{20}$ This replacement necessitates the formation of threefold coordinated oxygen atoms in order to maintain the glass stoichiometry, and leads to an increase with density in the number of small rings. The increase when $\rho / \rho_{0}>1.16$ in the number of threefold rings (i.e. those containing $3 \mathrm{Ge}$ and $3 \mathrm{O}$ atoms), which are attributed to the $D_{2}$ band at $\simeq 520 \mathrm{~cm}^{-1}$ in Raman spectra, ${ }^{198,199}$ is consistent with the measured density dependence of this feature. ${ }^{200-202}$ A preference is shown for sixfold rings when $\rho / \rho_{0}=1$ and for fourfold rings when $\rho / \rho_{0}=1.64$.

Like $\mathrm{GeO}_{2}, \mathrm{SiO}_{2}$ glass also forms under ambient conditions a network of corner-sharing $\mathrm{MO}_{4}$ tetrahedra where the mean intertetrahedral $\mathrm{M}-\mathrm{O}-\mathrm{M}$ bond angle is $151^{\circ}$ for $\mathrm{SiO}_{2}$ as compared to $132(2)^{\circ}$ for $\mathrm{GeO}_{2} \cdot{ }^{71,203}$ With increasing density, $\mathrm{SiO}_{2}$ is also converted from a tetrahedral to an octahedral glass, the changes occurring at higher pressures by comparison to $\mathrm{GeO}_{2} \cdot{ }^{200-202}$ As shown in Fig. 16, the structural changes do, however, occur at

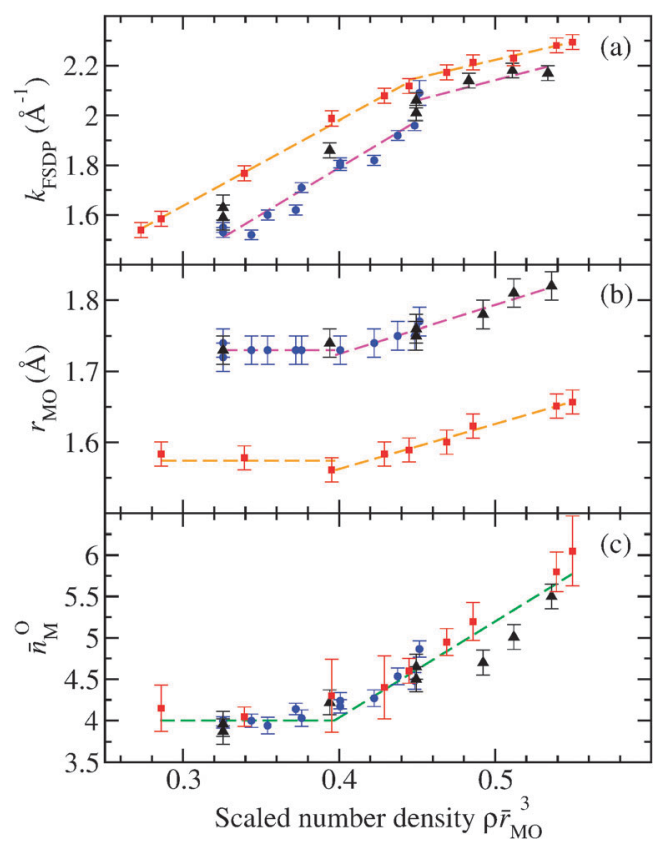

Fig. 16 The dependence on the scaled number-density $\rho \bar{r}_{\mathrm{MO}}{ }^{3}$ of several parameters describing the compressed structures of $\mathrm{GeO}_{2}$ and $\mathrm{SiO}_{2}$ glass, where $\bar{r}_{\mathrm{MO}}$ is the $\mathrm{M}-\mathrm{O}$ bond length at ambient pressure and $\mathrm{M}$ denotes either $\mathrm{Ge}$ or $\mathrm{Si}^{61}$ (a) The position of the first sharp diffraction peak $k_{\text {FSDP }}$ in the measured total structure factors, (b) the bond distance $r_{\mathrm{MO}}$ as deduced from the position of the first peak in the total pair-distribution functions, and (c) the coordination number $\bar{n}_{\mathrm{M}}^{\mathrm{O}}$. In the expression $\rho \bar{r}_{\mathrm{MO}}{ }^{3}$ the values $\bar{r}_{\mathrm{GeO}}=1.73 \AA$ and $\bar{r}_{\mathrm{SiO}}=1.60 \AA$ were used. ${ }^{70}$ The neutron diffraction results for $\mathrm{GeO}_{2}$ were taken from Drewitt et al. ${ }^{60}$ ((blue) $\left.\mathbf{O}\right)$ and from Salmon et al. ${ }^{61}$ ((black) $\mathbf{\Delta}$ ) while the X-ray diffraction results for $\mathrm{SiO}_{2}$ were taken from Benmore et al. ${ }^{190}$ ((red) $\left.\mathbf{\square}\right)$. The broken straight lines are fits to the data sets at either small or large densities. Figure from Salmon et al. ${ }^{61}$ (C) IOP Publishing. Reproduced with permission. All rights reserved. similar scaled number densities $\rho \bar{r}_{\mathrm{MO}}{ }^{3}$ where $\bar{r}_{\mathrm{MO}}$ is the ambientpressure bond-length. ${ }^{61}$ For example, the measured $\mathrm{M}-\mathrm{O}$ bond lengths and coordination numbers for both $\mathrm{GeO}_{2}$ and $\mathrm{SiO}_{2}$ barely change at low pressures, but then increase once the dimensionless number density $\rho \bar{r}_{\mathrm{MO}}{ }^{3} \gtrsim 0.4$. For both glasses, there is also an alteration to the rate of change of the FSDP position $k_{\mathrm{FSDP}}$ with increasing density at $\rho \bar{r}_{\mathrm{MO}}{ }^{3} \simeq 0.45$.

In the case of $\mathrm{GeO}_{2}$ glass, the regime when $\rho \bar{r}_{\mathrm{MO}}{ }^{3} \lesssim 0.4$ is associated with a reorganization of $\mathrm{GeO}_{4}$ units, the regime when $0.4 \lesssim \rho \bar{r}_{\mathrm{MO}}{ }^{3} \lesssim 0.45$ corresponds to the replacement of $\mathrm{GeO}_{4}$ units by predominantly $\mathrm{GeO}_{5}$ units, and the regime when $\rho \bar{r}_{\mathrm{MO}}{ }^{3} \gtrsim 0.45$ corresponds to the appearance of a growing concentration of $\mathrm{GeO}_{6}$ units. According to Fig. 16, similar structural transformations are expected for $\mathrm{SiO}_{2}$ glass. Hence, $\mathrm{SiO}_{4}$ units will be replaced predominantly by $\mathrm{SiO}_{5}$ units in a dimensionless number density regime which corresponds to a pressure range from about 15 to $24 \mathrm{GPa}$, and appreciable concentrations of $\mathrm{SiO}_{6}$ units will begin to form at pressures in excess of $\sim 24 \mathrm{GPa}$. These findings are in broad agreement with the molecular dynamics studies of $\mathrm{SiO}_{2}$ by Liang et al. ${ }^{204}$ and $\mathrm{Wu}$ et al. ${ }^{205}$ From the experimental perspective, X-ray Raman scattering oxygen K-edge spectra show little change at pressures below $14 \mathrm{GPa}$ and may be interpreted at higher pressures in terms of the formation of $\mathrm{SiO}_{5}$ units. ${ }^{205,206}$ In addition, the appearance of $\mathrm{SiO}_{5}$ units at pressures above $12 \mathrm{GPa}$ has been suggested on the basis of X-ray Raman scattering silicon L-edge spectra. ${ }^{207}$

The packing fraction of oxygen atoms arranged in regular tetrahedral units is given by $\eta_{\mathrm{O}}=16 \sqrt{2} \pi \rho r_{\mathrm{MO}}{ }^{3} / 27 \sqrt{3}$ (see Table 1 ) and, as shown by Fig. 16, the nearest-neigbour bond length $\bar{r}_{\text {MO }}$ changes little between ambient and a scaled number density $\rho \bar{r}_{\mathrm{MO}}{ }^{3} \simeq 0.4$. The latter corresponds to a packing fraction $\eta_{\mathrm{O}} \simeq 0.61$, close to the value of $\simeq 0.64$ expected for a random close-packing of hard spheres. ${ }^{82}$ Thus, fivefold coordinated $\mathrm{Si}$ or Ge atoms begin to appear only when the oxygen atoms have filled the space that is available to them. $\dagger+\dagger \dagger$

As discussed in Section 5, glasses are not expected to give an accurate account of the structure of the corresponding liquids, especially for more fragile glass-forming systems. However, since glasses can explore localities on an energy landscape that are inaccessible to the crystalline state, the formation of fivefold coordinated silicon atoms at high pressures is likely to be mirrored in the liquid phases of e.g. compressed high-temperature liquid silicates. These materials are of geophysical relevance since they account for a significant proportion of the Earth's mantle, and structural changes that take place under high-pressure and temperature conditions alter physical properties (e.g. the compressibility, thermal expansivity and viscosity) that govern magma related processes such as volcanic activity and the differentiation and evolution in composition of the planetary interior. ${ }^{208,209}$ As suggested by molecular dynamics simulations, ${ }^{210,211}$ the formation

ttt† Since the volume of a regular tetrahedron $V_{\text {tet }}=(8 / 9 \sqrt{3}) r_{\mathrm{MO}}{ }^{3}$ and the number density of tetrahedral units for $\mathrm{SiO}_{2}$ or $\mathrm{GeO}_{2}$ at low pressures $\rho_{\text {tet }}=\rho / 3$, the packing fraction for tetrahedral units is $\eta_{\text {tet }}=(24 / 9 \sqrt{3}) \rho r_{\mathrm{MO}}{ }^{3}$. The appearance of fivefold coordinated $\mathrm{Si}$ or Ge atoms at $\rho \bar{r}_{\mathrm{MO}}{ }^{3} \simeq 0.4$ therefore coincides with a packing fraction for tetrahedral units of $\simeq 0.62$. 
of $\mathrm{SiO}_{5}$ species in these liquids is likely to have a substantial impact on the extent of network polymerisation and, thereby, the physical properties of the melt.

\section{Quantum effects in the hydrogen bonded network of water}

Oxygen is a ubiquitous element, ${ }^{212}$ playing an essential role in most scientific and technological disciplines, and is often incorporated within a structurally disordered material where examples include molten silicates in planetary science, ${ }^{213}$ glasses used for lasers and optical communications, ${ }^{5}$ the insulating oxide layers in silicon-based electronic devices, ${ }^{214}$ and water in biological processes. ${ }^{7,8}$ To investigate the possibility of using oxygen as a site specific structural probe in neutron diffraction experiments, the sensitive technique of neutron interferometry ${ }^{215}$ has recently been applied to measure the coherent scattering length values of the isotopes ${ }^{17} \mathrm{O}$ and ${ }^{18} \mathrm{O}$ relative to oxygen of natural isotopic abundance ${ }^{\text {nat }} \mathrm{O} .{ }^{216}$ The measured values of $b_{17} \mathrm{O}=5.867(4)$ and $b_{18} \mathrm{O}=6.009(5) \mathrm{fm}$ give a factor of $\simeq 6$ increase in the scattering length contrast between ${ }^{18} \mathrm{O}$ and ${ }^{\text {nat }} \mathrm{O}$ by comparison with standard tabulations. ${ }^{217,218}$

These results encouraged Zeidler et al..$^{21-23}$ to make the first application of the method of oxygen NDIS to measure the structure of light $\left(\mathrm{H}_{2} \mathrm{O}\right)$ versus heavy $\left(\mathrm{D}_{2} \mathrm{O}\right)$ water to investigate the role played by nuclear quantum effects, such as zero point energy and tunnelling, on water's hydrogen bonded network. These quantum effects manifest themselves by differences between the structure and dynamics of light versus heavy water which lead to changes in properties such as their melting and boiling points, their temperature of maximum density, and their interaction with biological systems. ${ }^{8,219-222}$ In addition, techniques such as two-dimensional infra-red spectroscopy commonly require $\mathrm{H}$ to be partially substituted by $\mathrm{D}$, and it is therefore important to understand the accompanying effect on the structure and dynamics of water. ${ }^{223,224}$

Let diffraction experiments be made on light water samples of $\mathrm{H}_{2}{ }^{\text {nat }} \mathrm{O}$ and $\mathrm{H}_{2}{ }^{18} \mathrm{O}$ and on heavy water samples of $\mathrm{D}_{2}{ }^{\text {nat }} \mathrm{O}$ and $\mathrm{D}_{2}{ }^{18} \mathrm{O}$. In each case, the neutron and proton/deuteron masses are comparable, which leads to a large contribution to the measured diffraction patterns from inelastic scattering effects, for which there is no exact theory. ${ }^{17,225,226}$ In experiments on water, it is therefore desirable to minimise the distortion that these unwanted effects have on the measured diffraction patterns, and this can be achieved by using the oxygen NDIS method. For example, in the case of light water it follows from eqn (1) that subtraction of the measured differential scattering cross-sections gives the expression

$$
\Delta F_{\mathrm{H}}^{\prime}(k) \equiv \mathrm{d} \sigma /\left.\mathrm{d} \Omega\right|_{\mathrm{H}} ^{18}-\mathrm{d} \sigma /\left.\mathrm{d} \Omega\right|_{\mathrm{H}} ^{\text {nat }}=\Delta F_{\mathrm{H}}(k)+\Delta P_{\mathrm{H}}(k)
$$

where the first-difference function is given by

$$
\begin{aligned}
\Delta F_{\mathrm{H}}(k)= & F_{\mathrm{H}}^{18}(k)-F_{\mathrm{H}}^{\text {nat }}(k) \\
= & c_{\mathrm{O}}{ }^{2}\left(b_{18}{ }^{2}-b_{\text {nat }}{ }^{2}\right)\left[S_{\mathrm{OO}}(k)-1\right] \\
& +2 c_{\mathrm{O}} c_{\mathrm{H}} b_{\mathrm{H}}\left(b_{18}-b_{\text {nat }^{O}}\right)\left[S_{\mathrm{OH}}(k)-1\right] .
\end{aligned}
$$

The $\mathrm{H}-\mathrm{H}$ correlations are thereby eliminated and, since $\mathrm{H}$ nuclei are lighter than $\mathrm{O}$ nuclei and twice as numerous, the main contributions arising from inelastic scattering are also eliminated to leave a residual inelasticity correction $\Delta P_{\mathrm{H}}(k)$. In the case of heavy water, subtraction of the measured differential scattering cross-sections gives

$$
\Delta F_{\mathrm{D}}^{\prime}(k) \equiv \mathrm{d} \sigma /\left.\mathrm{d} \Omega\right|_{\mathrm{D}} ^{18}-\mathrm{d} \sigma /\left.\mathrm{d} \Omega\right|_{\mathrm{D}} ^{\text {nat }}=\Delta F_{\mathrm{D}}(k)+\Delta P_{\mathrm{D}}(k)
$$

where the first-difference function is given by

$$
\begin{aligned}
\Delta F_{\mathrm{D}}(k)= & F_{\mathrm{D}}^{18}(k)-F_{\mathrm{D}}^{\text {nat }}(k) \\
= & c_{\mathrm{O}}{ }^{2}\left(b_{18}{ }^{2}-b_{\text {nat }^{\circ}}^{2}\right)\left[S_{\mathrm{OO}}(k)-1\right] \\
& +2 c_{\mathrm{O}} c_{\mathrm{D}} b_{\mathrm{D}}\left(b_{18} \mathrm{O}-b_{\text {nat } \mathrm{O}}\right)\left[S_{\mathrm{OD}}(k)-1\right]
\end{aligned}
$$

and $\Delta P_{\mathrm{D}}(k)$ is a residual inelasticity correction. The corresponding real-space functions $\Delta G_{\mathrm{H}}(r)$ and $\Delta G_{\mathrm{D}}(r)$ are obtained by substituting $S_{\alpha \beta}(k)$ for $g_{\alpha \beta}(r)$ in eqn (28) and (30), respectively.

The complexity of correlations associated with a total structure factor can also be simplified by forming the second-difference function

$$
\begin{aligned}
\Delta F_{\mathrm{OX}}(k) & =1+\frac{\Delta F_{\mathrm{D}}^{\prime}(k)-\Delta F_{\mathrm{H}}^{\prime}(k)-\Delta P_{\mathrm{OX}}(k)}{2 c_{\mathrm{O}} c_{\mathrm{H}}\left(b_{\mathrm{D}}-b_{\mathrm{H}}\right)\left(b_{18} \mathrm{O}-b_{\text {nat }}\right)} \\
& =1+\frac{\Delta F_{\mathrm{D}}(k)-\Delta F_{\mathrm{H}}(k)}{2 c_{\mathrm{O}} c_{\mathrm{H}}\left(b_{\mathrm{D}}-b_{\mathrm{H}}\right)\left(b_{18} \mathrm{O}-b_{\text {nat } \mathrm{O}}\right)}
\end{aligned}
$$

where $\mathrm{X}$ represents a weighted average of $\mathrm{H}$ and D. $\Delta F_{\mathrm{OX}}(k)$ corresponds to $S_{\mathrm{OD}}(k)$ (or $S_{\mathrm{OH}}(k)$ ) if the net quantum effects are sufficiently small that there is good cancellation of the $S_{\mathrm{OO}}(k)$ functions for light and heavy water and $S_{\mathrm{OH}}(k) \simeq S_{\mathrm{OD}}(k)$. The residual inelasticity correction $\Delta P_{\mathrm{Ox}}(k)=\Delta P_{\mathrm{D}}(k)-\Delta P_{\mathrm{H}}(k)$ is particularly small because the inelasticity corrections on the first-difference functions have the same sign and are comparable in magnitude. ${ }^{23}$

The measured first-difference functions are shown in Fig. 17(a) and the corresponding real-space functions are shown in Fig. 18(a), where the robustness of these results was thoroughly checked by using the procedures described in ref. 23. The first peak in $\Delta G_{\mathrm{D}}(r)$ at 0.985(5) $\AA$ and the first trough in $\Delta G_{\mathrm{H}}(r)$ at $0.990(5) \AA$ arise from intra-molecular $\mathrm{O}-\mathrm{D}$ and $\mathrm{O}-\mathrm{H}$ correlations and give coordination numbers of $\bar{n}_{\mathrm{O}}^{\mathrm{D}}=1.9(1)$ and $\bar{n}_{\mathrm{O}}^{\mathrm{H}}=2.0(2)$. The O-D distance compares with a value of 0.983(8) ^ obtained by Powles $^{225}$ from a re-analysis of neutron diffraction data for liquid heavy water at $294 \mathrm{~K}$, and with a value of 0.985(7) $\AA$ as estimated from a pair-distribution function analysis of neutron diffraction data for ice- $\mathrm{I}_{\mathrm{h}} \cdot{ }^{228}$ The difference between the O-D and $\mathrm{O}-\mathrm{H}$ bond lengths of $\simeq 0.5 \%$ compares to $\simeq 0.4 \%$ for both the vapour phase of water in its ground vibrational state (where the O-D and $\mathrm{O}-\mathrm{H}$ distances are 0.9687 and $0.9724 \AA$, respectively) ${ }^{229}$ and for ice- $\mathrm{I}_{\mathrm{h}}$ at $123 \mathrm{~K}$ (where the $\mathrm{O}-\mathrm{D}$ and $\mathrm{O}-\mathrm{H}$ distances are $0.983(5)$ and $0.987(5) \AA$, respectively). ${ }^{230}$ All of these values are smaller than the bond-length difference of $\sim 3 \%$ found in the neutron and X-ray diffraction investigation of liquid water by Soper and Benmore ${ }^{231}$ using the EPSR method, a difference that is much larger than found by any 


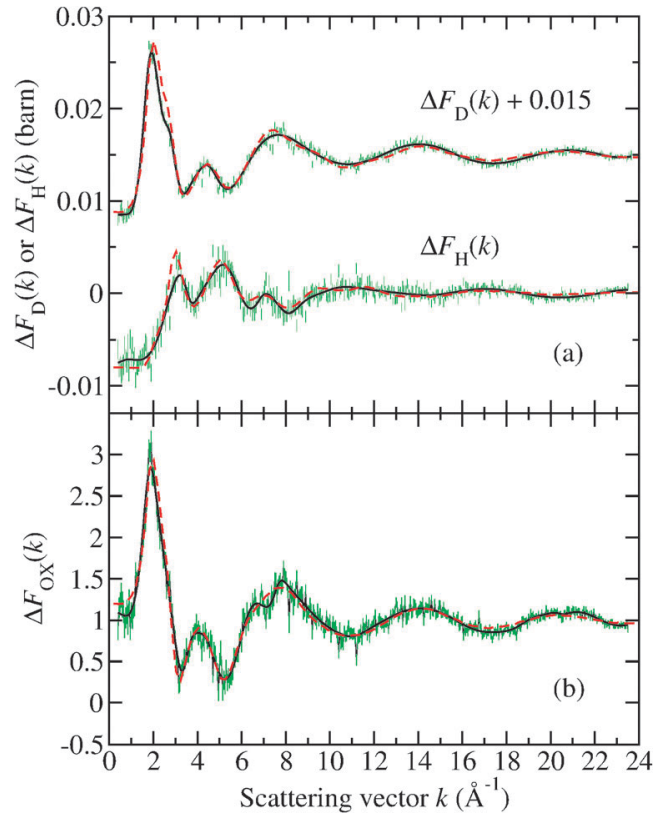

Fig. 17 (a) The first-difference functions $\Delta F_{\mathrm{D}}(k) /$ barn $=0.0059(2)\left[S_{\mathrm{OD}}(k)-1\right]+$ $0.00262(8)\left[S_{\mathrm{OO}}(k)-1\right]$ and $\Delta F_{\mathrm{H}}(k) /$ barn $=-0.0033(1)\left[\mathrm{S}_{\mathrm{OH}}(k)-1\right]+$ $0.00263(8)\left[S_{\mathrm{OO}}(k)-1\right]$ and $(\mathrm{b})$ the second-difference function $\Delta F_{\mathrm{OX}}(k)$ which is constructed by subtracting $\Delta F_{\mathrm{H}}(k)$ from $\Delta F_{\mathrm{D}}(k)$ and is equal to $S_{\mathrm{OD}}(k)$ (or $\mathrm{S}_{\mathrm{OH}}(k)$ ) if the net quantum effects are sufficiently small (see the text). ${ }^{21,23}$ In (a) and (b) the vertical bars give the statistical errors on the measured data points and the solid (black) curves give spline fits that were used to generate the corresponding realspace functions shown in Fig. 18. The broken (red) curves show the results obtained from path integral molecular dynamics using the flexible anharmonic TTM3-F model for water. ${ }^{227}$ Reprinted with permission from Zeidler et al. ${ }^{21}$ Copyright (2011) by the American Physical Society.

theoretical prediction. ${ }^{21,23}$ Indeed, in more recent work Soper reports a revised bond-length difference that is a factor of ten smaller. ${ }^{232}$ 拥

Isotope effects in water have been investigated by McBride et al. ${ }^{234}$ by constructing a new model for $\mathrm{D}_{2} \mathrm{O}$ in which the $\mathrm{O}-\mathrm{H}$ bond length in the rigid and non-polarisable TIP4PQ/2005 water model was shortened in line with the bond-length difference reported by Zeidler et al. ${ }^{21,23}$ The isotope shifts found for the melting point temperature of ice- $\mathrm{I}_{\mathrm{h}}$, and for the temperature of maximum density of the liquid, are in good agreement with the experimental results for $\mathrm{H}_{2} \mathrm{O}$ versus $\mathrm{D}_{2} \mathrm{O}$.

The measured second-difference function $\Delta F_{\mathrm{Ox}}(k)$ is shown in Fig. 17(b) and the corresponding real-space function $\Delta G_{\mathrm{OX}}(r)$ is shown in Fig. 18(b). The first peak in $\Delta G_{\text {OX }}(r)$ at $0.987(5) \AA$ is attributed to intra-molecular $\mathrm{O}-\mathrm{X}$ correlations and gives a coordination number $\bar{n}_{\mathrm{O}}^{\mathrm{X}}=2.0(1)$. The second peak at 1.83(2) $\AA$ corresponds to the inter-molecular $\mathrm{O}-\mathrm{X}$ hydrogen bond and is at the same position as the inter-molecular O-D peak position in $\Delta G_{\mathrm{D}}(r)$.

拥 In ref. 232 the EPSR method was used with the TIP4P-2005 reference potential model for water in order to improve agreement with X-ray diffraction data for the first inter-molecular O-O peak (A. K. Soper, private communication). The sensitivity of the EPSR method to the choice of reference potential is discussed elsewhere. ${ }^{22,23,94,233}$

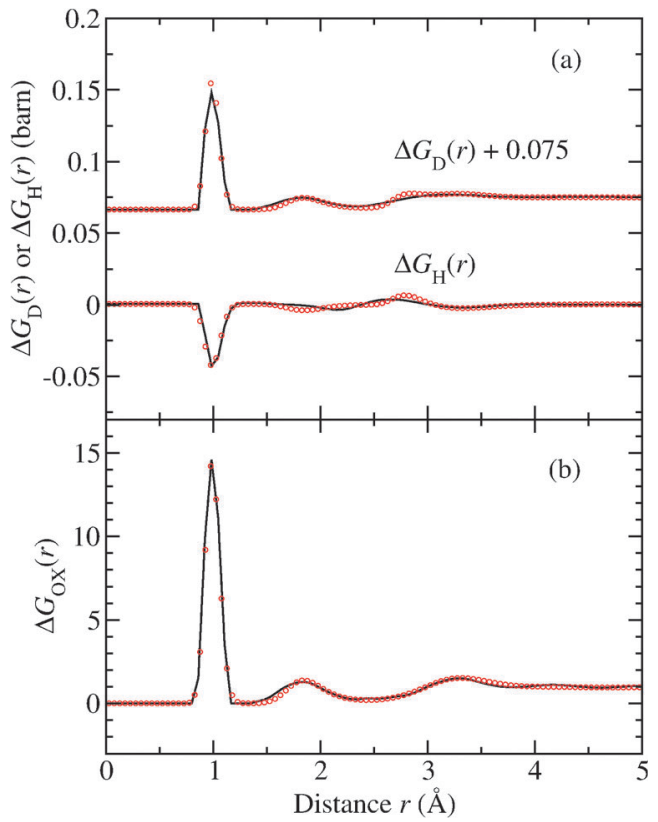

Fig. 18 (a) The first-difference functions $\Delta G_{D}(r) /$ barn $=0.0059(2)\left[g_{\mathrm{OD}}(r)-1\right]+$ $0.00262(8)\left[g_{\mathrm{OO}}(r)-1\right]$ and $\Delta G_{\mathrm{H}}(r) /$ barn $=-0.0033(1)\left[g_{\mathrm{OH}}(r)-1\right]+$ $0.00263(8)\left[g_{O O}(r)-1\right]$. The $\mathrm{O}-\mathrm{H}$ correlations in $\Delta G_{H}(r)$ have a negative weighting factor because $b_{\mathrm{H}}<0$. (b) The second-difference function $\Delta G_{\mathrm{OX}}(r)$ which is equal to $g_{\mathrm{OD}}(r)$ (or $\left.g_{\mathrm{OH}}(r)\right)$ if the net quantum effects are sufficiently small (see the text). In (a) and (b) the solid curves were obtained by Fourier transforming the spline fitted data sets presented in Fig. 17 before and after the application of a Lorch modification function. The data obtained from the first procedure were joined with the data obtained from the second procedure after the first peak or trough. The open circles show the results obtained from path integral molecular dynamics using the flexible anharmonic TTM3-F model for water. ${ }^{227}$ Reprinted with permission from Zeidler et al. ${ }^{21}$ Copyright (2011) by the American Physical Society.

The first- and second-difference functions were compared ${ }^{21,23}$ to the results obtained from path integral molecular dynamics simulations $^{235}$ of light and heavy water using several different models of water, namely the rigid models TIP4P and SPC/E, ${ }^{236,237}$ the flexible harmonic models SPC/F and $\mathrm{qSPC} / \mathrm{Fw},{ }^{238,239}$ and the flexible anharmonic models q-TIP4P/F and TTM3-F. ${ }^{227,240}$ These calculations, in which a system of quantum mechanical particles is mapped onto a simulation of classical ring polymers, provide an exact treatment of nuclear quantum fluctuations in the structure of a given potential energy model for water. $^{235}$ In the cases of rigid or flexible harmonic classical models, the introduction of quantum effects leads, in general, to a large de-structuring of the hydrogen bonded network as shown by (i) a decrease in height of the first peak in $g_{\mathrm{oo}}(r)$ which is an indicator of inter-molecular ordering, and (ii) an increase in the number of hydrogen bonds broken. ${ }^{239,241-243}$ This de-structuring can have dramatic implications for the dynamics with the diffusion coefficient changing by as much as $50 \% .^{239,240,244}$ Replication of the first O-O peak height reduction by using classical models requires an increase of the liquid temperature by $\Delta T \simeq 35-40 \mathrm{~K}$. By contrast, flexible anharmonic models and a recent first-principles molecular dynamics study predict much smaller changes in the structure of water, ${ }^{220,240}$ corresponding to $\Delta T \simeq 5-18 \mathrm{~K}$. 
Of the various models, the intra-molecular $\mathrm{O}-\mathrm{H}$ and $\mathrm{O}-\mathrm{D}$ bond length difference of $0.0054 \AA$ or $\simeq 0.5 \%$ found using the TTM3-F model is in excellent agreement with the new diffraction data, and the comparison in Fig. 18(a) shows that theory and experiment are essentially superposed in the region where the intra-molecular correlations dominate. The TTM3-F model gives inter-molecular $\mathrm{O}-\mathrm{D}$ and $\mathrm{O}-\mathrm{H}$ peak positions both equal to $1.84 \AA$, with weighted peak positions $\left\langle r_{\text {OX }}\right\rangle=$ $\int \mathrm{d} r r g_{\mathrm{OX}}(r) / \int \mathrm{d} r g_{\mathrm{OX}}(r)$ (where $\mathrm{X}$ denotes $\mathrm{D}$ or $\mathrm{H}$ ) both equal to $1.91 \AA$ A i.e. the average length of a hydrogen bond barely changes between light and heavy water. The TTM3-F model reproduces accurately the $\mathrm{O}-\mathrm{H}$ stretching region of the infra-red absorption spectrum of liquid water, ${ }^{227}$ and when quantum effects are included the diffusion coefficient $D_{\mathrm{qm}}=0.95 \times 0.237=$ $0.225 \AA^{2} \mathrm{ps}^{-1}$ is in excellent accord with the measured value of $0.229 \AA^{2} \mathrm{ps}^{-1}$. $^{227,240}$

As discussed by Zeidler et al., ${ }^{21,23}$ the quantitative agreement found between the oxygen NDIS experimental results and the path integral molecular dynamics results for the TTM3-F model supports the proposal of "competing quantum effects" in liquid water. ${ }^{220,240}$ In this hypothesis inter-molecular zero point energy and tunnelling weaken the hydrogen bond network, as previously predicted, ${ }^{239,241-243}$ but quantum fluctuations in the anharmonic intra-molecular $\mathrm{O}-\mathrm{H}$ bond increase its length and hence the dipole moment of each water molecule. This higher dipole acts, in turn, to increase the binding between molecules and hence the net effect of quantum fluctuations is smaller than originally suggested from rigid water simulations. It can be shown that, for a selection of water models, the extent of cancellation is strongly related to $\Delta r=r_{\mathrm{OH}}-r_{\mathrm{OD}}$, the change observed in the intra-molecular bond length on substituting $\mathrm{H}$ for $\mathrm{D}$ in the path integral simulations. ${ }^{21,23}$ The largest values of $\Delta r$ correspond to flexible anharmonic models which show a much smaller change upon including quantum effects in (i) the O-O peak height, (ii) the number of hydrogen bonds broken, and (iii) the diffusion coefficient. In the case of the TTM3-F model, the cancellation between opposing quantum effects is large, leading to a small fall in the $\mathrm{O}-\mathrm{O}$ peak height of 0.03 and a ratio of quantum to classical diffusion coefficients of 0.95 . These changes are much smaller than for rigid models which cannot exhibit any competition between intra-molecular and inter-molecular quantum contributions. Support for the proposal of competing quantum effects also comes from calculations of the isotope fractionation ratio i.e. the equilibrium $\mathrm{D} / \mathrm{H}$ ratio for the liquid versus the vapour phases of water. ${ }^{245}$ In this case, the flexible anharmonic q-TIP4P/F water model predicts the correct magnitude for the isotope fractionation ratio, but the TTM3-F water model overestimates the extent of the intra-molecular versus inter-molecular competition.

A large cancellation of competing quantum effects is fortuitous for experimental methods that exploit H/D isotope substitution such as the method of neutron diffraction with H/D isotope substitution,,$^{94,246-248}$ two-dimensional infra-red spectroscopy ${ }^{223,224}$ and nuclear magnetic resonance spectroscopy. ${ }^{249}$ This is because when these methods are used to study systems involving either light or heavy water at $300 \mathrm{~K}$ the structural environments probed, as well as the quantum contribution to the dynamics, are likely to be similar.

\section{Conclusions and outlook}

The results presented in this Perspective demonstrate that, by matching carefully designed experiments with suitable neutron diffraction instrumentation, it is possible to use NDIS to probe the detailed structure of liquids and glasses on multiple length scales. Moreover, it is now possible to apply the NDIS method even when the samples are small, as in high-pressure Paris-Edinburgh press experiments ${ }^{20}$ and in high-temperature aerodynamic-levitation experiments, ${ }^{18}$ or when the neutron scattering length contrast is small, as in experiments on water using oxygen isotope substitution. ${ }^{21-23}$ The different length scales that were discussed are associated with e.g. (i) intermediate versus extended-range ordering in network glass-forming systems, where there is a competition that affects the relative fragility of a material; (ii) changes in the local structural motifs on vitrification in the fragile glass-former $\mathrm{CaAl}_{2} \mathrm{O}_{4}$, which are accompanied by the development of extended branched-chains of Ca-centred polyhedra; and (iii) intra-molecular versus inter-molecular ordering in liquid water, where there is a competition between the associated quantum effects.

The results obtained from carefully performed NDIS experiments play an important role in helping to assess e.g. the accuracy of various theoretical schemes that are employed to make atomistic models for different classes of disordered materials. This is illustrated by (i) first-principles investigations of liquid and glassy chalcogenide materials at ambient pressure, where the progression from use in self-consistent calculations of the local density approximation (LDA) to a generalised gradient approximation using the Becke-Lee-Yang-Parr (BLYP) functional to describe the electron exchange and correlation, has led to models that are in better agreement with the NDIS and other experimental results; ${ }^{29,66-68,107-110,250}$ (ii) classical molecular dynamics simulations of network-forming oxide glasses, where the parameterisation of a polarisable ion model for $\mathrm{GeO}_{2}$ using $a b$ initio results has led to transferrable potentials that can be used to accurately predict, for the first time, the material properties under high-pressure conditions; ${ }^{20,28,36}$ and (iii) path integral molecular dynamics investigations of liquid water, where flexible anharmonic models best account for the measured difference between the intra-molecular $\mathrm{O}-\mathrm{H}$ and $\mathrm{O}-\mathrm{D}$ bond distances in light and heavy water, ${ }^{21,23}$ thus giving support to the hypothesis of competing intra-molecular versus intermolecular quantum effects. ${ }^{220,240}$ Once the correct theoretical scheme has been established, these simulations can be used to enrich the information about a material that can be extracted, and they can also be used to predict the changes in material properties that occur when e.g. new chemical species are added or the state conditions are varied.

In summary, the NDIS method can be used to investigate the coordination environment of a growing number of chemical species under a variety of different sample conditions. This is enabling an increased exploitation of the neutron's attributes 
as a structural probe to study a broad range of disordered materials having scientific and/or technological significance. The development of new high counting-rate instrumentation at central neutron scattering facilities for diffraction studies of liquid and glassy materials, which can often be adapted for making a pair-distribution function analysis of the structure of crystalline or nano-crystalline materials, will continue to offer new opportunities for the NDIS method to provide benchmark experimental results.

\section{Acknowledgements}

We would particularly like to thank Henry Fischer at the Institut Laue-Langevin (ILL, Grenoble) for his help and advice with many of the NDIS experiments reported in this manuscript. Special thanks also go to Adrian Barnes (Bristol), Wilson Crichton (ESRF), Gabriel Cuello (ILL), Louis Hennet (Orlèans) and Stefan Klotz (Paris) for their contributions to the experimental work; and to Sandro Jahn (Potsdam), Sébastien Le Roux (Strasbourg), Tom Markland (Stanford), Dario Marrocchelli (Dublin), Carlo Massobrio (Strasbourg), Alfredo Pasquarello (Lausanne) and Mark Wilson (Oxford) for their contributions on the molecular dynamics front. Pierre Chieux (ILL), John Enderby (Bristol), Bob Evans (Bristol), Paul Madden (Oxford) and Shin'ichi Takeda (Kyushu) have all offered sound advice over the years. Much of the NDIS work described in this Perspective has benefitted immensely from the contributions made by past and present group members, including Ian Penfold, Chris Benmore (Argonne National Laboratory), Ingrid Petri, Takeshi Usuki (Yamagata), Richard Martin (Aston), James Drewitt (Edinburgh), Prae Chirawatkul (Synchrotron Light Research Institute, Thailand), Dean Whittaker (JET, Culham), Kamil Wezka and Harry Bone. We also thank James Drewitt for his help in preparing Fig. 1. The support of the EPSRC (Grant Ref.: EP/J009741/1) and ILL is gratefully acknowledged.

\section{References}

1 N. E. Cusack, The Physics of Structurally Disordered Matter, Hilger, Bristol, 1987.

2 S. R. Elliott, Physics of Amorphous Materials, Longman, Harlow, 2nd edn, 1990.

3 A. Feltz, Amorphous Inorganic Materials and Glasses, VCH, Weinheim, 1993.

4 CRC Handbook of Laser Science and Technology (Suppl. 2), ed. M. J. Weber, CRC Press, Boca Raton, FL, 1995.

5 M. Yamane and Y. Asahara, Glasses for Photonics, Cambridge University Press, Cambridge, 2000.

6 G. N. Greaves and S. Sen, Adv. Phys., 2007, 56, 1.

7 F. Franks, Water: A Matrix of Life, Royal Society of Chemistry, Cambridge, 2nd edn, 2000.

8 F. Paesani and G. A. Voth, J. Phys. Chem. B, 2009, 113, 5702.

9 P. S. Salmon, Nat. Mater., 2002, 1, 87.

10 P. Bondot, Acta Crystallogr., Sect. A: Cryst. Phys., Diffr., Theor. Gen. Cryst., 1974, 30, 470.

11 J. E. Enderby, D. M. North and P. A. Egelstaff, Philos. Mag., 1966, 14, 961.
12 S. Ramos, A. C. Barnes, G. W. Neilson and M. J. Capitan, Chem. Phys., 2000, 258, 171.

13 S. Hosokawa, W.-C. Pilgrim, J.-F. Bérar and S. Kohara, Phys. Status Solidi A, 2011, 208, 2544.

14 A. C. Barnes, S. B. Lague, M. A. Hamilton, H. E. Fischer, A. N. Fitch and E. Dooryhee, J. Phys.: Condens. Matter, 1998, 10, L645.

15 D. L. Price, M.-L. Saboungi and A. C. Barnes, Phys. Rev. Lett., 1998, 81, 3207.

16 S. Sampath, C. J. Benmore, K. M. Lantzky, J. Neuefeind, K. Leinenweber, D. L. Price and J. L. Yarger, Phys. Rev. Lett., 2003, 90, 115502.

17 H. E. Fischer, A. C. Barnes and P. S. Salmon, Rep. Prog. Phys., 2006, 69, 233.

18 J. W. E. Drewitt, L. Hennet, A. Zeidler, S. Jahn, P. S. Salmon, D. R. Neuville and H. E. Fischer, Phys. Rev. Lett., 2012, 109, 235501.

19 L. B. Skinner, C. J. Benmore, J. K. R. Weber, S. Tumber, L. Lazareva, J. Neuefeind, L. Santodonato, J. Du and J. B. Parise, J. Phys. Chem. B, 2012, 116, 13439.

20 K. Wezka, P. S. Salmon, A. Zeidler, D. A. J. Whittaker, J. W. E. Drewitt, S. Klotz, H. E. Fischer and D. Marrocchelli, J. Phys.: Condens. Matter, 2012, 24, 502101.

21 A. Zeidler, P. S. Salmon, H. E. Fischer, J. C. Neuefeind, J. M. Simonson, H. Lemmel, H. Rauch and T. E. Markland, Phys. Rev. Lett., 2011, 107, 145501.

22 A. Zeidler, P. S. Salmon, H. E. Fischer, J. C. Neuefeind, J. M. Simonson, H. Lemmel, H. Rauch and T. E. Markland, Phys. Rev. Lett., 2012, 108, 259604.

23 A. Zeidler, P. S. Salmon, H. E. Fischer, J. C. Neuefeind, J. M. Simonson and T. E. Markland, J. Phys.: Condens. Matter, 2012, 24, 284126.

24 M. Wilson and P. A. Madden, J. Phys.: Condens. Matter, 1993, 5, 6833.

25 P. A. Madden and M. Wilson, Chem. Soc. Rev., 1996, 25, 339.

26 F. Hutchinson, A. J. Rowley, M. K. Walters, M. Wilson, P. A. Madden, J. C. Wasse and P. S. Salmon, J. Chem. Phys., 1999, 111, 2028.

27 S. Jahn and P. A. Madden, Phys. Earth Planet. Inter., 2007, $162,129$.

28 D. Marrocchelli, M. Salanne, P. A. Madden, C. Simon and P. Turq, Mol. Phys., 2009, 107, 443.

29 C. Massobrio, Lect. Notes Phys., 2010, 795, 343.

30 R. L. McGreevy and L. Pusztai, Mol. Simul., 1988, 1, 359.

31 R. L. McGreevy, J. Phys.: Condens. Matter, 2001, 13, R877.

32 A. K. Soper, Chem. Phys., 1996, 202, 295.

33 A. K. Soper, Phys. Rev. B: Condens. Matter Mater. Phys., 2005, 72, 104204.

34 Y. Liang, C. R. Miranda and S. Scandolo, High-Pressure Res.: Appl. Geophys., 2008, 28, 35.

35 C. Massobrio, M. Celino, P. S. Salmon, R. A. Martin, M. Micoulaut and A. Pasquarello, Phys. Rev. B: Condens. Matter Mater. Phys., 2009, 79, 174201.

36 D. Marrocchelli, M. Salanne and P. A. Madden, J. Phys.: Condens. Matter, 2010, 22, 152102.

37 C. A. Angell, Science, 1995, 267, 1924. 
38 R. Evans, R. J. F. Leote de Carvalho, J. R. Henderson and D. C. Hoyle, J. Chem. Phys., 1994, 100, 591.

39 R. J. F. Leote de Carvalho and R. Evans, Mol. Phys., 1994, 83, 619.

40 P. S. Salmon, J. Phys.: Condens. Matter, 2006, 18, 11443.

41 T. E. Faber and J. M. Ziman, Philos. Mag., 1965, 11, 153.

42 E. Lorch, J. Phys. C: Solid State Phys., 1969, 2, 229.

43 A. K. Soper and E. R. Barney, J. Appl. Crystallogr., 2011, 44, 714 .

44 A. B. Bhatia and D. E. Thornton, Phys. Rev. B: Solid State, 1970, 2, 3004.

45 P. S. Salmon, Proc. R. Soc. London, Ser. A, 1992, 437, 591.

46 J. Blétry, Z. Naturforsch., A: Phys. Phys. Chem. Kosmophys., 1976, 31, 960.

47 A. B. Bhatia and V. K. Ratti, Phys. Chem. Liq., 1977, 6, 201.

48 M. Maret, P. Chieux, J. M. Dubois and A. Pasturel, J. Phys.: Condens. Matter, 1991, 3, 2801.

49 V. Bitrián, J. Trullàs and M. Silbert, Physica B, 2008, 403, 4249. 50 H. Ruppersberg and H. Egger, J. Chem. Phys., 1975, 63, 4095.

51 A. Zeidler, J. W. E. Drewitt, P. S. Salmon, A. C. Barnes, W. A. Crichton, S. Klotz, H. E. Fischer, C. J. Benmore, S. Ramos and A. C. Hannon, J. Phys.: Condens. Matter, 2009, 21, 474217.

52 K. F. Ludwig Jr, W. K. Warburton, L. Wilson and A. I. Bienenstock, J. Chem. Phys., 1987, 87, 604.

53 A. Zeidler, P. S. Salmon, R. A. Martin, T. Usuki, P. E. Mason, G. J. Cuello, S. Kohara and H. E. Fischer, Phys. Rev. B: Condens. Matter Mater. Phys., 2010, 82, 104208.

54 H. E. Fischer, G. J. Cuello, P. Palleau, D. Feltin, A. C. Barnes, Y. S. Badyal and J. M. Simonson, Appl. Phys. A: Solid Surf., 2002, 74, S160.

55 A. K. Soper, in Advanced Neutron Sources - Inst. Phys. Conf. Ser., ed. D. K. Hyer, Institute of Physics, Bristol, 1989, vol. 97, p. 353.

56 A. C. Hannon, Nucl. Instrum. Methods Phys. Res., Sect. A, 2005, 551, 88.

57 D. T. Bowron, A. K. Soper, K. Jones, S. Ansell, S. Birch, J. Norris, L. Perrott, D. Riedel, N. J. Rhodes, S. R. Wakefield, A. Botti, M.-A. Ricci, F. Grazzi and M. Zoppi, Rev. Sci. Instrum., 2010, 81, 033905.

58 J. Neuefeind, K. K. Chipley, C. A. Tulk, J. M. Simonson and M. J. Winokur, Physica B, 2006, 385-386, 1066.

59 T. Hattori, Y. Katayama, A. Machida, T. Otomo and K. Suzuya, J. Phys.: Conf. Ser., 2010, 215, 012024.

60 J. W. E. Drewitt, P. S. Salmon, A. C. Barnes, S. Klotz, H. E. Fischer and W. A. Crichton, Phys. Rev. B: Condens. Matter Mater. Phys., 2010, 81, 014202.

61 P. S. Salmon, J. W. E. Drewitt, D. A. J. Whittaker, A. Zeidler, K. Wezka, C. L. Bull, M. G. Tucker, M. C. Wilding, M. Guthrie and D. Marrocchelli, J. Phys.: Condens. Matter, 2012, 24, 415102.

62 A. Zeidler, J. Appl. Crystallogr., 2012, 45, 122.

63 A. K. Soper, G. W. Neilson, J. E. Enderby and R. A. Howe, J. Phys. C: Solid State Phys., 1977, 10, 1793.

64 P. S. Salmon, S. Xin and H. E. Fischer, Phys. Rev. B: Condens. Matter Mater. Phys., 1998, 58, 6115.
65 F. G. Edwards, J. E. Enderby, R. A. Howe and D. I. Page, J. Phys. C: Solid State Phys., 1975, 8, 3483.

66 (a) I. T. Penfold and P. S. Salmon, Phys. Rev. Lett., 1991, 67, 97; (b) I. T. Penfold and P. S. Salmon, Phys. Rev. Lett., 1992, 68, 253.

67 P. S. Salmon and I. Petri, J. Phys.: Condens. Matter, 2003, 15, S1509.

68 I. Petri, P. S. Salmon and H. E. Fischer, Phys. Rev. Lett., 2000, 84, 2413.

69 P. S. Salmon, R. A. Martin, P. E. Mason and G. J. Cuello, Nature, 2005, 435, 75.

70 P. S. Salmon, A. C. Barnes, R. A. Martin and C. J. Cuello, Phys. Rev. Lett., 2006, 96, 235502.

71 P. S. Salmon, A. C. Barnes, R. A. Martin and G. J. Cuello, J. Phys.: Condens. Matter, 2007, 19, 415110.

72 P. S. Salmon, J. Phys.: Condens. Matter, 2007, 19, 455208.

73 S. Biggin and J. E. Enderby, J. Phys. C: Solid State Phys., 1981, 14, 3129.

74 Q. Mei, C. J. Benmore, S. Sen, R. Sharma and J. L. Yarger, Phys. Rev. B: Condens. Matter Mater. Phys., 2008, 78, 144204.

75 M. Wilson and P. S. Salmon, Phys. Rev. Lett., 2009, 103, 157801.

76 S. C. Moss and D. L. Price, in Physics of Disordered Materials, ed. D. Adler, H. Fritzsche and S. R. Ovshinsky, Plenum, New York, 1985, p. 77.

77 S. R. Elliott, Nature, 1991, 354, 445.

78 P. S. Salmon, Proc. R. Soc. London, Ser. A, 1994, 445, 351.

79 P. S. Salmon, J. Non-Cryst. Solids, 2007, 353, 2959.

80 P. S. Salmon, J. Phys.: Condens. Matter, 2005, 17, S3537.

81 J. A. E. Desa, A. C. Wright, J. Wong and R. N. Sinclair, J. Non-Cryst. Solids, 1982, 51, 57.

82 A. V. Anikeenko and N. N. Medvedev, Phys. Rev. Lett., 2007, 98, 235504.

83 M. J. L. Sangster and M. Dixon, Adv. Phys., 1976, 25, 247.

84 N. C. Pyper, in Adv. Solid State Chem., ed. C. R. A. Catlow, JAI Press, London, 1991, vol. 2, p. 223.

85 R. Kjellander and B. Forsberg, J. Phys. A: Math. Gen., 2005, 38, 5405.

86 V. A. Levashov, S. J. L. Billinge and M. F. Thorpe, Phys. Rev. B: Condens. Matter Mater. Phys., 2005, 72, 024111.

87 J. K. Christie, S. N. Taraskin and S. R. Elliott, Phys. Rev. B: Condens. Matter Mater. Phys., 2004, 70, 134207.

88 J. K. Christie, S. N. Taraskin and S. R. Elliott, J. Phys.: Condens. Matter, 2004, 16, S5109.

89 M. Wilson, Phys. Chem. Chem. Phys., 2012, 14, 12701.

90 V. Petkov and D. Le Messurier, J. Phys.: Condens. Matter, 2010, 22, 115402.

91 S. B. Mamedov, N. D. Aksenov, L. L. Makarov and Y. F. Batrakov, J. Non-Cryst. Solids, 1996, 195, 272.

92 P. Boolchand and W. J. Bresser, Philos. Mag. B, 2000, 80, 1757.

93 A. K. Soper, J. Phys.: Condens. Matter, 2007, 19, 415108.

94 A. K. Soper, J. Phys.: Condens. Matter, 2007, 19, 335206.

95 M. Cobb, D. A. Drabold and R. L. Cappelletti, Phys. Rev. B: Condens. Matter Mater. Phys., 1996, 54, 12162.

96 M. Cobb and D. A. Drabold, Phys. Rev. B: Condens. Matter Mater. Phys., 1997, 56, 3054. 
97 C. Massobrio, A. Pasquarello and R. Car, Phys. Rev. Lett., 1998, 80, 2342.

98 C. Massobrio, A. Pasquarello and R. Car, J. Am. Chem. Soc., 1999, 121, 2943.

99 C. Massobrio, A. Pasquarello and R. Car, Comput. Mater. Sci., 2000, 17, 115.

100 X. Zhang and D. A. Drabold, Phys. Rev. B: Condens. Matter Mater. Phys., 2000, 62, 15695.

101 C. Massobrio, A. Pasquarello and R. Car, Phys. Rev. B: Condens. Matter Mater. Phys., 2001, 64, 144205.

102 C. Massobrio, M. Celino and A. Pasquarello, Phys. Rev. B: Condens. Matter Mater. Phys., 2004, 70, 174202.

103 C. Massobrio and A. Pasquarello, J. Phys.: Condens. Matter, 2007, 19, 415111.

104 C. Massobrio and A. Pasquarello, Phys. Rev. B: Condens. Matter Mater. Phys., 2007, 75, 014206.

105 C. Massobrio and A. Pasquarello, Phys. Rev. B: Condens. Matter Mater. Phys., 2008, 77, 144207.

106 M. Wilson, B. K. Sharma and C. Massobrio, J. Chem. Phys., 2008, 128, 244505.

107 M. Micoulaut, R. Vuilleumier and C. Massobrio, Phys. Rev. B: Condens. Matter Mater. Phys., 2009, 79, 214205.

108 C. Massobrio, M. Micoulaut and P. S. Salmon, Solid State Sci., 2010, 12, 199.

109 M. Micoulaut, S. Le Roux and C. Massobrio, J. Chem. Phys., 2012, 136, 224504.

110 A. Bouzid and C. Massobrio, J. Chem. Phys., 2012, 137, 046101.

111 E. L. Gjersing, S. Sen and B. G. Aitken, J. Phys. Chem. C, 2010, 114, 8601.

112 S. Sen and Z. Gan, J. Non-Cryst. Solids, 2010, 356, 1519.

113 M. Kibalchenko, J. R. Yates, C. Massobrio and A. Pasquarello, J. Phys. Chem. C, 2011, 115, 7755.

114 T. G. Edwards, S. Sen and E. L. Gjersing, J. Non-Cryst. Solids, 2012, 358, 609.

115 P. Vashishta, R. K. Kalia, G. A. Antonio and I. Ebbsjö, Phys. Rev. Lett., 1989, 62, 1651.

116 P. Vashishta, R. K. Kalia and I. Ebbsjö, Phys. Rev. B: Condens. Matter Mater. Phys., 1989, 39, 6034.

117 H. Iyetomi, P. Vashishta and R. K. Kalia, Phys. Rev. B: Condens. Matter Mater. Phys., 1991, 43, 1726.

118 P. Vashishta, R. K. Kalia, J. P. Rino and I. Ebbsjö, Phys. Rev. B: Condens. Matter Mater. Phys., 1990, 41, 12197.

119 P. Chirawatkul, A. Zeidler, P. S. Salmon, S. Takeda, Y. Kawakita, T. Usuki and H. E. Fischer, Phys. Rev. B: Condens. Matter Mater. Phys., 2011, 83, 014203.

120 H. Fujii, S. Tahara, Y. Kato, S. Kohara, M. Itou, Y. Kawakita and S. Takeda, J. Non-Cryst. Solids, 2007, 353, 2094.

121 S. Takeda, H. Fujii, Y. Kawakita, Y. Kato, S. Fujita, Y. Yokota and S. Kohara, Mater. Sci. Eng., A, 2007, 449-451, 590.

122 S. Takeda, H. Fujii, Y. Kawakita, S. Tahara, S. Nakashima, S. Kohara and M. Itou, J. Alloys Compd., 2008, 452, 149.

123 I. Egry and D. Holland-Moritz, Eur. Phys. J.: Spec. Top., 2011, 196, 131.

124 D. Ma, A. D. Stoica and X.-L. Wang, Nat. Mater., 2009, 8, 30. 125 W. Klement Jr, R. H. Willens and P. Duwez, Nature, 1960, 187, 869.
126 H. S. Chen and D. Turnbull, J. Appl. Phys., 1967, 38, 3646. 127 V. N. Novikov and A. P. Sokolov, Phys. Rev. B: Condens. Matter Mater. Phys., 2006, 74, 064203.

128 W. H. Wang, Prog. Mater. Sci., 2007, 52, 540.

129 D. B. Miracle, Nat. Mater., 2004, 3, 697.

130 W. L. Johnson, JOM, 2002, 54, 40.

131 J. F. Löffler, Intermetallics, 2003, 11, 529.

132 A. Inoue and A. Takeuchi, Acta Mater., 2011, 59, 2243.

133 E. Axinte, Mater. Des., 2012, 35, 518.

134 H. W. Sheng, W. K. Luo, F. M. Alamgir, J. M. Bai and E. Ma, Nature, 2006, 439, 419.

135 P. F. Guan, T. Fujita, A. Hirata, Y. H. Liu and M. W. Chen, Phys. Rev. Lett., 2012, 108, 175501.

136 L. Yang, G. Q. Guo, L. Y. Chen, C. L. Huang, T. Ge, D. Chen, P. K. Liaw, K. Saksl, Y. Ren, Q. S. Zeng, B. LaQua, F. G. Chen and J. Z. Jiang, Phys. Rev. Lett., 2012, 109, 105502.

137 P. A. Egelstaff, N. H. March and N. C. McGill, Can. J. Phys., 1974, 52, 1651.

138 J. Chihara, J. Phys. F: Met. Phys., 1987, 17, 295.

139 S. Takeda, M. Inui, S. Tamaki, K. Maruyama and Y. Waseda, J. Phys. Soc. Jpn., 1994, 63, 1794.

140 S. Takeda, Y. Kawakita, M. Inui, K. Maruyama, S. Tamaki and Y. Waseda, J. Non-Cryst. Solids, 1996, 205-207, 365.

141 P. S. Salmon, I. Petri, P. H. K. de Jong, P. Verkerk, H. E. Fischer and W. S. Howells, J. Phys.: Condens. Matter, 2004, 16, 195.

142 T. Freltoft, J. K. Kjems and S. K. Sinha, Phys. Rev. B: Condens. Matter Mater. Phys., 1986, 33, 269.

143 P. Mangin, G. Marchal, C. Mourey and C. Janot, Phys. Rev. B: Condens. Matter Mater. Phys., 1980, 21, 3047.

144 C. Grodon, M. Dijkstra, R. Evans and R. Roth, Mol. Phys., 2005, 103, 3009.

145 P. Hopkins and M. Schmidt, J. Phys.: Condens. Matter, 2010, 22, 325108.

146 D. L. Price, High-Temperature Levitated Materials, Cambridge University Press, Cambridge, 2010.

147 J. K. R. Weber, J. J. Felten, B. Cho and P. C. Nordine, Nature, 1998, 393, 769.

148 L. B. Skinner, A. C. Barnes and W. Crichton, J. Phys.: Condens. Matter, 2006, 18, L407.

149 D. Holland-Moritz, T. Schenk, P. Convert, T. Hansen and D. M. Herlach, Meas. Sci. Technol., 2005, 16, 372.

150 L. Hennet, I. Pozdnyakova, A. Bytchkov, V. Cristiglio, P. Palleau, H. E. Fischer, G. J. Cuello, M. Johnson, P. Melin, D. Zanghi, S. Brassamin, J.-F. Brun, D. L. Price and M.-L. Saboungi, Rev. Sci. Instrum., 2006, 77, 053903.

151 (a) A. C. Barnes, L. B. Skinner, P. S. Salmon, A. Bytchkov, I. Pozdnyakova, T. O. Farmer and H. E. Fischer, Phys. Rev. Lett., 2009, 103, 225702; (b) A. C. Barnes, L. B. Skinner, P. S. Salmon, A. Bytchkov, I. Pozdnyakova, T. O. Farmer and H. E. Fischer, Phys. Rev. Lett., 2011, 106, 119602.

152 T. Voigtmann, A. Meyer, D. Holland-Moritz, S. Stüber, T. Hansen and T. Unruh, Europhys. Lett., 2008, 82, 66001.

153 D. Holland-Moritz, S. Stüber, H. Hartmann, T. Unruh, T. Hansen and A. Meyer, Phys. Rev. B: Condens. Matter Mater. Phys., 2009, 79, 064204. 
154 T. Schenk, V. Simonet, D. Holland-Moritz, R. Bellissent, T. Hansen, P. Convert and D. M. Herlach, Europhys. Lett., 2004, 65, 34 .

155 J. E. Drewitt, S. Jahn, V. Cristiglio, A. Bytchkov, M. Leydier, S. Brassamin, H. E. Fischer and L. Hennet, J. Phys.: Condens. Matter, 2011, 23, 155101.

156 J. Akola, S. Kohara, K. Ohara, A. Fujiwara, Y. Watanabe, A. Masuno, T. Usuki, T. Kubo, A. Nakahira, K. Nitta, T. Uruga, J. K. R. Weber and C. J. Benmore, Proc. Natl. Acad. Sci. U. S. A., 2013, 110, 10129, DOI: 10.1073/pnas.1300908110.

157 D. Iuga, C. Morais, Z. Gan, D. R. Neuville, L. Cormier and D. Massiot, J. Am. Chem. Soc., 2005, 127, 11540.

158 S. Y. Park and S. K. Lee, Geochim. Cosmochim. Acta, 2012, 80, 125.

159 J. F. Stebbins and D. Sykes, Am. Mineral., 1990, 75, 943.

160 G. S. Henderson, Can. Mineral., 2005, 43, 1921.

161 W. E. Jackson, F. Farges, M. Yeager, P. A. Mabrouk, S. Rossano, G. A. Waychunas, E. I. Solomon and G. E. Brown Jr, Geochim. Cosmochim. Acta, 2005, 69, 4315.

162 G. S. Henderson, G. Calas and J. F. Stebbins, Elements, 2006, 2, 269.

163 J. F. Stebbins, E. V. Dubinsky, K. Kanehashi and K. E. Kelsey, Geochim. Cosmochim. Acta, 2008, 72, 910.

164 D. Giordano and D. B. Dingwell, J. Phys.: Condens. Matter, 2003, 15, S945.

165 D. Giordano and J. K. Russell, J. Phys.: Condens. Matter, 2007, 19, 205148.

166 D. Giordano, J. K. Russell and D. B. Dingwell, Earth Planet. Sci. Lett., 2008, 271, 123.

167 C. J. Benmore and P. S. Salmon, Phys. Rev. Lett., 1994, 73, 264.

168 J. Liu and P. S. Salmon, Europhys. Lett., 1997, 39, 521.

169 P. S. Salmon and S. Xin, Phys. Rev. B: Condens. Matter Mater. Phys., 2002, 65, 064202.

170 R. A. Martin, P. S. Salmon, H. E. Fischer and G. J. Cuello, Phys. Rev. Lett., 2003, 90, 185501.

171 R. A. Martin, P. S. Salmon, H. E. Fischer and G. J. Cuello, J. Phys.: Condens. Matter, 2003, 15, 8235.

172 I. T. Penfold and P. S. Salmon, Phys. Rev. Lett., 1990, 64, 2164.

173 S. A. van der Aart, P. Verkerk, A. C. Barnes, P. S. Salmon, R. Winter, H. Fischer, L. A. de Graaf and W. van der Lugt, Physica B, 1998, 241-243, 961.

174 J. C. Wasse and P. S. Salmon, J. Phys.: Condens. Matter, 1999, 11, 1381.

175 J. C. Wasse, P. S. Salmon and R. G. Delaplane, J. Phys.: Condens. Matter, 2000, 12, 9539.

176 J. C. Wasse, I. Petri and P. S. Salmon, J. Phys.: Condens. Matter, 2001, 13, 6165.

177 R. A. Martin, P. S. Salmon, C. J. Benmore, H. E. Fischer and G. J. Cuello, Phys. Rev. B: Condens. Matter Mater. Phys., 2003, 68, 054203.

178 R. A. Martin, P. S. Salmon, H. E. Fischer and G. J. Cuello, J. Non-Cryst. Solids, 2004, 345-346, 208.

179 S. Xin, J. Liu and P. S. Salmon, Phys. Rev. B: Condens. Matter Mater. Phys., 2008, 78, 064207.
180 K. Wezka, A. Zeidler, P. S. Salmon, P. Kidkhunthod, A. C. Barnes and H. E. Fischer, J. Non-Cryst. Solids, 2011, 357, 2511.

181 V. V. Brazhkin and A. G. Lyapin, J. Phys.: Condens. Matter, 2003, 15, 6059.

182 M. C. Wilding, M. Wilson and P. F. McMillan, Chem. Soc. Rev., 2006, 35, 964.

183 G. N. Greaves, M. C. Wilding, S. Fearn, D. Langstaff, F. Kargl, S. Cox, Q. Vu Van, O. Majérus, C. J. Benmore, R. Weber, C. M. Martin and L. Hennet, Science, 2008, 322, 566.

184 D. Daisenberger, T. Deschamps, B. Champagnon, M. Mezouar, R. Q. Cabrera, M. Wilson and P. F. McMillan, J. Phys. Chem. B, 2011, 115, 14246.

185 M. Guthrie, C. A. Tulk, C. J. Benmore, J. Xu, J. L. Yarger, D. D. Klug, J. S. Tse, H.-K. Mao and R. J. Hemley, Phys. Rev. Lett., 2004, 93, 115502.

186 M. Wilding, M. Guthrie, C. L. Bull, M. G. Tucker and P. F. McMillan, J. Phys.: Condens. Matter, 2008, 20, 244122.

187 E. Soignard, C. J. Benmore and J. L. Yarger, Rev. Sci. Instrum., 2010, 81, 035110.

188 S. Klotz, Techniques in High Pressure Neutron Scattering, CRC Press, Boca Raton, 2013.

189 G. Lelong, L. Cormier, G. Ferlat, V. Giordano, G. S. Henderson, A. Shukla and G. Calas, Phys. Rev. B: Condens. Matter Mater. Phys., 2012, 85, 134202.

190 C. J. Benmore, E. Soignard, S. A. Amin, M. Guthrie, S. D. Shastri, P. L. Lee and J. L. Yarger, Phys. Rev. B: Condens. Matter Mater. Phys., 2010, 81, 054105.

191 Q. Mei, C. J. Benmore, R. T. Hart, E. Bychkov, P. S. Salmon, C. D. Martin, F. M. Michel, S. M. Antao, P. J. Chupas, P. L. Lee, S. D. Shastri, J. B. Parise, K. Leinenweber, S. Amin and J. L. Yarger, Phys. Rev. B: Condens. Matter Mater. Phys., 2006, 74, 014203.

192 M. Guthrie, C. A. Tulk, J. Molaison and A. M. dos Santos, Phys. Rev. B: Condens. Matter Mater. Phys., 2012, 85, 184205.

193 M. Micoulaut, J. Phys.: Condens. Matter, 2004, 16, L131.

194 M. Micoulaut, Y. Guissani and B. Guillot, Phys. Rev. E: Stat. Phys., Plasmas, Fluids, Relat. Interdiscip. Top., 2006, 73, 031504.

195 K. V. Shanavas, N. Garg and S. M. Sharma, Phys. Rev. B: Condens. Matter Mater. Phys., 2006, 73, 094120.

196 T. Li, S. Huang and J. Zhu, Chem. Phys. Lett., 2009, 471, 253. 197 X. F. Zhu and L. F. Chen, Physica B, 2009, 404, 4178.

198 L. Giacomazzi, P. Umari and A. Pasquarello, Phys. Rev. Lett., 2005, 95, 075505.

199 L. Giacomazzi, P. Umari and A. Pasquarello, Phys. Rev. B: Condens. Matter Mater. Phys., 2006, 74, 155208.

200 D. J. Durben and G. H. Wolf, Phys. Rev. B: Condens. Matter Mater. Phys., 1991, 43, 2355.

201 C. H. Polsky, K. H. Smith and G. H. Wolf, J. Non-Cryst. Solids, 1999, 248, 159.

202 M. Micoulaut, L. Cormier and G. S. Henderson, J. Phys.: Condens. Matter, 2006, 18, R753.

203 M. G. Tucker, D. A. Keen, M. T. Dove and K. Trachenko, J. Phys.: Condens. Matter, 2005, 17, S67.

204 Y. Liang, C. R. Miranda and S. Scandolo, Phys. Rev. B: Condens. Matter Mater. Phys., 2007, 75, 024205. 
205 M. Wu, Y. Liang, J.-Z. Jiang and J. S. Tse, Sci. Rep., 2012, 2, 398.

206 J.-F. Lin, H. Fukui, D. Prendergast, T. Okuchi, Y. Q. Cai, N. Hiraoka, C.-S. Yoo, A. Trave, P. Eng, H. Y. Hu and P. Chow, Phys. Rev. B: Condens. Matter Mater. Phys., 2007, 75, 012201.

207 H. Fukui, M. Kanzaki, N. Hiraoka and Y. Q. Cai, Phys. Rev. B: Condens. Matter Mater. Phys., 2008, 78, 012203.

208 J.-P. Poirier, Introduction to the Physics of the Earth's Interior, Cambridge University Press, Cambridge, 2nd edn, 2000.

209 D. L. Anderson, New Theory of the Earth, Cambridge University Press, Cambridge, 2007.

210 C. A. Angell, P. A. Cheeseman and S. Tamaddon, Science, 1982, 218, 885.

211 J. D. Kubicki and A. C. Lasaga, Phys. Chem. Miner., 1991, 17, 661.

212 N. Lane, Oxygen: The Molecule that Made the World, Oxford University Press, Oxford, 2002.

213 M. Mookherjee, L. Stixrude and B. Karki, Nature, 2008, 452, 983.

214 A. Pasquarello, M. S. Hybertsen and R. Car, Nature, 1998, 396, 58.

215 H. Rauch and S. A. Werner, Neutron Interferometry: Lessons in Experimental Quantum Mechanics, Clarendon Press, Oxford, 2000.

216 H. E. Fischer, J. M. Simonson, J. C. Neuefeind, H. Lemmel, H. Rauch, A. Zeidler and P. S. Salmon, J. Phys.: Condens. Matter, 2012, 24, 505105.

217 L. Koester, H. Rauch and E. Seymann, At. Data Nucl. Data Tables, 1991, 49, 65.

218 V. F. Sears, Neutron News, 1992, 3, 26.

219 B. Tomberli, C. J. Benmore, P. A. Egelstaff, J. Neuefeind and V. Honkimäki, J. Phys.: Condens. Matter, 2000, $12,2597$.

220 B. Chen, I. Ivanov, M. L. Klein and M. Parrinello, Phys. Rev. Lett., 2003, 91, 215503.

221 R. T. Hart, C. J. Benmore, J. Neuefeind, S. Kohara, B. Tomberli and P. A. Egelstaff, Phys. Rev. Lett., 2005, 94, 047801.

222 J. A. Morrone and R. Car, Phys. Rev. Lett., 2008, 101, 017801.

223 J. D. Eaves, J. J. Loparo, C. J. Fecko, S. T. Roberts, A. Tokmakoff and P. L. Geissler, Proc. Natl. Acad. Sci. U. S. A., 2005, 102, 13019.

224 J. J. Loparo, S. T. Roberts and A. Tokmakoff, J. Chem. Phys., 2006, 125, 194522.

225 J. G. Powles, Mol. Phys., 1981, 42, 757.
226 A. K. Soper, Mol. Phys., 2009, 107, 1667.

227 G. S. Fanourgakis and S. S. Xantheas, J. Chem. Phys., 2008, 128, 074506.

228 M. A. Floriano, D. D. Klug, E. Whalley, E. C. Svensson, V. F. Sears and E. D. Hallman, Nature, 1987, 329, 821.

229 R. L. Cook, F. C. De Lucia and P. Helminger, J. Mol. Spectrosc., 1974, 53, 62.

230 W. F. Kuhs and M. S. Lehmann, in Water Science Reviews 2, ed. F. Franks, Cambridge University Press, Cambridge, 1986, p. 1.

231 A. K. Soper and C. J. Benmore, Phys. Rev. Lett., 2008, 101, 065502.

232 A. K. Soper, ISRN Phys. Chem., 2013, 279463.

233 A. K. Soper and C. J. Benmore, Phys. Rev. Lett., 2012, 108, 259603.

234 C. McBride, J. L. Aragones, E. G. Noya and C. Vega, Phys. Chem. Chem. Phys., 2012, 14, 15199.

235 M. Parrinello and A. Rahman, J. Chem. Phys., 1984, 80, 860.

236 W. L. Jorgensen, J. Chandrasekhar, J. D. Madura, R. W. Impey and M. L. Klein, J. Chem. Phys., 1983, 79, 926.

237 H. J. C. Berendsen, J. R. Grigera and T. P. Straatsma, J. Phys. Chem., 1987, 91, 6269.

238 K. Toukan and A. Rahman, Phys. Rev. B: Condens. Matter Mater. Phys., 1985, 31, 2643.

239 F. Paesani, W. Zhang, D. A. Case, T. E. Cheatham III and G. A. Voth, J. Chem. Phys., 2006, 125, 184507.

240 S. Habershon, T. E. Markland and D. E. Manolopoulos, J. Chem. Phys., 2009, 131, 024501.

241 R. A. Kuharski and P. J. Rossky, J. Chem. Phys., 1985, 82, 5164.

242 A. Wallqvist and B. J. Berne, Chem. Phys. Lett., 1985, 117, 214.

243 L. Hernández de la Peña and P. G. Kusalik, J. Chem. Phys., 2004, 121, 5992.

244 L. Hernández de la Peña and P. G. Kusalik, J. Chem. Phys., 2006, 125, 054512.

245 T. E. Markland and B. J. Berne, Proc. Natl. Acad. Sci. U. S. A., 2012, 109, 7988.

246 A. K. Soper, Chem. Phys., 2000, 258, 121.

247 A. K. Soper and M. A. Ricci, Phys. Rev. Lett., 2000, 84, 2881.

248 J. C. Dore, M. Garawi and M.-C. Bellissent-Funel, Mol. Phys., 2004, 102, 2015.

249 X. Cui, S. Mao, M. Liu, H. Yuan and Y. Du, Langmuir, 2008, 24, 10771.

250 S. Le Roux, A. Zeidler, P. S. Salmon, M. Boero, M. Micoulaut and C. Massobrio, Phys. Rev. B: Condens. Matter Mater. Phys., 2011, 84, 134203. 